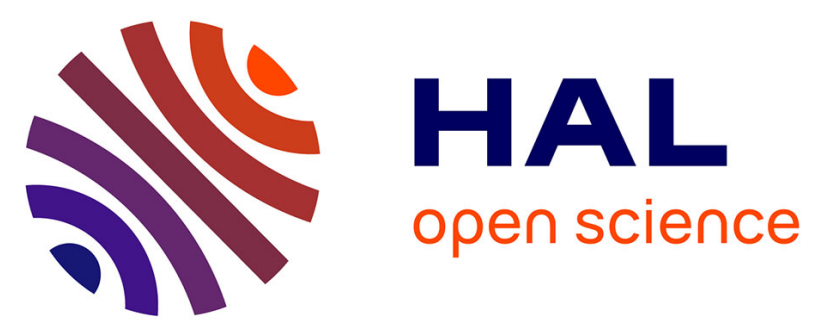

\title{
Structure and Energetics of Dye-Sensitized NiO Interfaces in Water from Ab Initio MD and Large-Scale GW Calculations
}

\author{
Alekos Segalina, Sébastien Lebègue, Dario Rocca, Simone Piccinin, \\ Mariachiara Pastore
}

\section{To cite this version:}

Alekos Segalina, Sébastien Lebègue, Dario Rocca, Simone Piccinin, Mariachiara Pastore. Structure and Energetics of Dye-Sensitized NiO Interfaces in Water from Ab Initio MD and LargeScale GW Calculations. Journal of Chemical Theory and Computation, 2021, 17 (8), pp.5225-5238. 10.1021/acs.jctc.1c00354 . hal-03420793

\section{HAL Id: hal-03420793 https://hal.science/hal-03420793}

Submitted on 9 Nov 2021

HAL is a multi-disciplinary open access archive for the deposit and dissemination of scientific research documents, whether they are published or not. The documents may come from teaching and research institutions in France or abroad, or from public or private research centers.
L'archive ouverte pluridisciplinaire HAL, est destinée au dépôt et à la diffusion de documents scientifiques de niveau recherche, publiés ou non, émanant des établissements d'enseignement et de recherche français ou étrangers, des laboratoires publics ou privés. 


\title{
Structure and Energetics of Dye-Sensitized $\mathrm{NiO}$ Interfaces in Water from ab-initio MD and Large-Scale GW Calculations
}

\author{
Alekos Segalina $^{a}$, Sébastien Lèbegue ${ }^{a}$, Dario Rocca ${ }^{a}$, \\ Simone Piccinin ${ }^{b, *}$ and Mariachiara Pastore ${ }^{a, *}$ \\ ${ }^{a}$ Université de Lorraine \& CNRS, \\ LPCT, UMR 7019, F-54000 Nancy, France \\ ${ }^{b}$ Consiglio Nazionale delle Ricerche, Istituto Officina dei Materiali, Trieste, Italy \\ July 9, 2021
}




\begin{abstract}
The energy level alignment across solvated molecule/semiconductor interfaces is a crucial property for the correct functioning of dye-sensitized photo-electrodes, where, following the absorption of solar light, a cascade of interfacial hole/electron transfer processes has to efficiently take place. In light of the difficulty of performing X-ray photoelectron spectroscopy measurements at the molecule/solvent/metal-oxide interface, being able to accurately predict the level alignment by first-principles calculations on realistic structural models would represent an important step toward the optimization of the device. In this respect dye/NiO surfaces, employed in p-type dye-sensitized solar cells, are undoubtedly challenging for $a b$ initio methods and, also for this reason, much less investigated than the n-type dye $/ \mathrm{TiO}_{2}$ counterpart. Here we consider the $\mathrm{C} 343$-sensitized NiO surface in water and combine ab initio Molecular Dynamics (AIMD) simulations with $\mathrm{GW}\left(\mathrm{G}_{0} \mathrm{~W}_{0}\right)$ calculations, performed along the MD trajectory, to reliably describe the structure and energetics of the interface when explicit solvation and finite temperature effects are accounted for. We show that the differential perturbative correction on the $\mathrm{NiO}$ and molecule states obtained at GW level is mandatory to recover the correct (physical) interfacial energetics, allowing hole transfer from the semiconductor valence band to the HOMO of the dye. Moreover, the calculated average driving force quantitatively agrees with the experimental estimate.
\end{abstract}

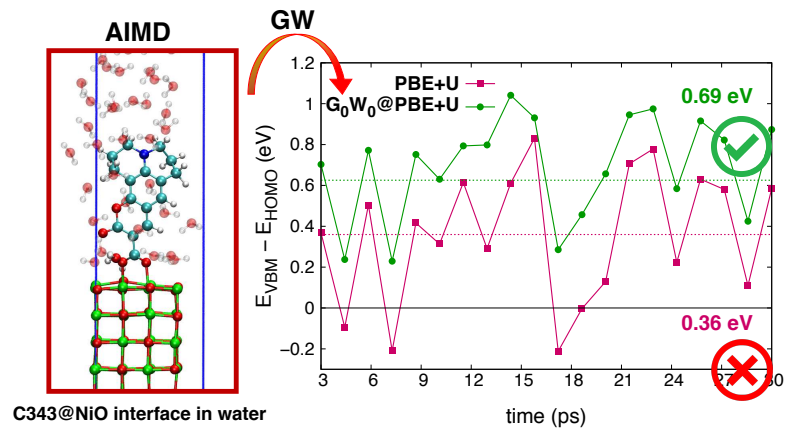

TOC 


\section{Introduction}

Thirty years have passed since the pioneering work of O'Regan and Grätzel ${ }^{1}$ paving the way to the development of efficient dye sensitized solar cells (DSSCs). The standard architecture of a DSSC includes the use of a single photoactive electrode that can be either a photoanode (n-type DSSCs) $)^{1,2}$ or a photocathode (p-type DSSCs). ${ }^{3,4}$ The photo-active interface in a DSSC is composed of three principal ingredients: a wide band gap semiconductor (SC), usually $\mathrm{TiO}_{2}$ and $\mathrm{NiO}$ in n- and p-type DSSCs, respectively, a dye sensitizer, either a full organic or a metallorganic compound, and a redox shuttle. The functioning mechanism of a $\mathrm{n}$-/p-type DSSC is the following: sunlight is absorbed by the photosensitizing dye and the photo-excited electrons/holes are injected in the conduction/valence band (CB/VB) of the SC, while the oxidized/reduced redox shuttle, having regenerated the dye ground state (GS), is in turn reduced/oxidized at the counter electrode, closing the circuit. ${ }^{5}$ Clearly, a multitude of factors interplay in determining the overall device efficiency, ${ }^{6}$ such as the opto-electronic properties of the isolated dyes and semiconductors, the structure of the dye/SC interface, the rate of regeneration of the dye by the redox mediator and the efficiency of electron/hole injection into the SC. The energetic prerequisite, however, to realize the cascade of electron/hole transfer processes described above is the correct alignment of the redox potentials (energy levels) between the dye, the SC and the electrolyte: the excited/ground state potential of the dye should be higher/lower of the $\mathrm{CB} / \mathrm{VB}$ edge of the $\mathrm{SC}$ to have electron/hole injection, while the oxidation/reduction potential of the electrolyte couple should be higher/lower of the ground/excited state oxidation potential of the dye to have the GS regeneration.

If, on one hand, n-type DSSCs have known a rapid development in the last decades, with reported power conversion efficiencies (PCEs) of about $14 \%,{ }^{2,7}$ the performance of p-type DSSCs remains, on the other hand, extremely poor $(\mathrm{PCE} \sim 2.5 \%) .{ }^{3,8,9}$ Research efforts in boosting the efficiency of p-type dye-sensitized photo-electrodes, for both DSSCs and dye-sensitized photoelectrochemical cells (DS-PECS), ${ }^{10,11}$ are motivated by the possibility of combining a photocathode and a photoanode in tandem devices, to surpass the theoretical Shockley-Queisser upper efficiency limit $(\sim 30 \%)$ of a single photoactive electrode solar cell. ${ }^{12,13}$ 
Despite the recent encouraging attempts to find alternative transparent p-type semiconductors, ${ }^{10,14,15} \mathrm{NiO}$ is still the standard material, both in solar cells and photoelectrochemical cells. Mesoporous $\mathrm{NiO}$ displays good electrical conductivity, ${ }^{16,17}$ and recent works have reported ultrafast interfacial hole injection from photo-excited dyes. ${ }^{18-21}$ This material, however, shows poor interfacial charge transfer resistance and features trap states placed above the VB edge, which promote detrimental hole/electron recombination at the SC/dye/electrolyte interface. ${ }^{16,21-23}$

As a matter of fact, a detailed understanding and characterization of the energetics, trapping mechanisms and hole dynamics at the dye/NiO interface is still lacking, while this would be needed to give clear guidelines to optimise the structure of dyes, electrolytes and catalysts. ${ }^{24,25}$ In this regard, computational modeling is a powerful tool to predict the opto-electronic and redox properties of semiconductors, chromophores and catalysts as well as to provide atomistic insights on the structure, energetics and charge generation in model dye-sensitized interfaces. $^{26-35}$

As far as dye-sensitized $\mathrm{NiO}$ is concerned, however, relatively few computational works, based on density functional theory (DFT) calculations, have been reported. ${ }^{36-42}$ A common outcome of these studies is that the interfacial energetics, dictating the driving force for hole injection from the dye to the $\mathrm{NiO} \mathrm{VB}$, turns out to be extremely sensitive to the dye anchoring mode and solvation effects, thus indicating that the structural and electronic properties of complex interface between dye, SC and electrolyte play a major role in the properties of DSSCs. Recently, we studied, by means of ab-initio molecular dynamics (AIMD) and DFT calculations, ${ }^{36}$ the electronic structure of the $(100) \mathrm{NiO}$ surface sensitized by the Coumarin $\mathrm{C} 343$ dye. ${ }^{43-45}$ We found that the inclusion of explicit solvation effects is essential to predict a favorable energy level alignment to allow the hole transfer from the dye HOMO to the NiO VB states. The driving forces computed using both the PBE+U approach and the HSE06 hybrid functional were found, however, to be somewhat underestimated with respect to experiments, as a result of a sizeable destabilization of the dye's HOMO. As also reported for dye@ $\mathrm{TiO}_{2}$ systems, ${ }^{46,47}$ this suggests that so-called global hybrid functionals, which approximate the inverse dielectric function with a constant, are unable to correctly reproduce the interfacial energy level alignment 
in hybrid organic/inorganic interfaces, since molecules and SC surfaces may require different percentage of non-local exchange to be properly treated. ${ }^{46}$ This is especially true for the hybridized SC-dye interfacial levels, as they may require a screening being intermediate between those optimal for the dye and for the metal oxide slab. Coordinate-dependent hybrid functionals, developed using Green's functions formalism, have been recently introduced to account for the spatial dependence of the electron-electron correlation in hybrid materials. ${ }^{48,49}$ These functionals are based on the assumption that the screened Coulomb interaction can be approximated through a spatial-dependent local dielectric function and have been only recently applied to heterogeneous systems with good results. ${ }^{48-50}$ However, this approximation, as all DFT based methods, neglect dynamical polarization or image charge effects, which can be rather pronounced for the dye electronic states in the dye@SC interface and can critically influence the energy level alignment. ${ }^{46,55-58}$ These higher-order effects, as well as the spatial dependence of the electronic correlation, can be accounted for by using more refined approximations, such as many body perturbation theory (MBPT) based methods, where the dynamical polarization response to the addition or to the removal of a particle are rigorously described. $27,57,59,60$ In particular, within the MBPT methods, the Hedin's GW approximation ${ }^{61}$ is the state-of-the-art for the computational modeling of photoemission and inverse photoemission processes for solid systems, as well as for the calculation of ionization potentials (IP) and electron affinities (EA) of molecules. ${ }^{59,62,63}$ As a matter of fact, the high computational cost, unfavorable $\mathrm{O}\left(\mathrm{N}^{4}\right)$ scaling, and convergence problems of GW methods have hindered their routine application, even if, in the last decades, the fast development in computing power along with recent efficient code implementations ${ }^{64-67}$ have made it possible to run GW calculations for rather complex systems with hundreds of atoms. As we shall discuss later, when dealing with 2-dimensional materials, however, the convergence of the results with respect to the k-points grid, the GW and planewave cutoffs, the vacuum space and the number of empty bands remains critical and need to be carefully addressed. ${ }^{68-71}$ Here we report a theoretical characterization of the C343-sensitized $\mathrm{NiO}$ surface in water, by combining AIMD simulations with $\mathrm{GW}\left(\mathrm{G}_{0} \mathrm{~W}_{0}\right)$ calculations along the MD trajectory, to reliably describe the structure and energetics of the interface when explicit solvation and finite temperature effects are accounted for. As far as we know, this is the 
first time that large scale GW calculations are combined with AIMD simulations to study a realistic dye/water/SC interface, since similar approaches have been only reported for solvated semiconductor slabs. ${ }^{50}$ The present results show that the differential perturbative correction on the $\mathrm{NiO}$ and molecule states obtained at GW level is essential to recover the correct interfacial energetics. Notably, the GW driving forces calculated along the MD trajectory are always positive (i.e. dye HOMO below the $\mathrm{NiO}$ VBM) and the resulting average value, being double the value obtained by DFT+U and hybrid functionals, ${ }^{36}$ quantitatively agree with the experimental estimations. We believe that the relevance of these results is twofold: i) for the theoretical and computational community, we contribute to the fundamental knowledge necessary to simulate, through non-adiabatic MD, the photoinduced hole transfer process in dye sensitized-NiO photocathodes, which requires an accurate description of the interfacial energy level alignment; $51-54$ ii) for the experimental community, we have set up a computational protocol capable to provide reliable estimation of the hole injection driving forces, thus overcoming the difficulty of performing X-ray photoelectron spectroscopy (XPS) measurements at the molecule/solvent/metaloxide interface.

The paper is organized as follows: methods and models are presented in section 2; GW calculations in gas phase on the $\mathrm{NiO}$ bulk and isolated (100) $\mathrm{NiO}$ slab and C343 dye are discussed in section 3.1, where the convergence of the GW results with respect to the vacuum space and the number of empty bands is also evaluated; the electronic structure of the dye/NiO interface in vacuo is discussed in section 3.2, while the results obtained on the AIMD simulation in water are presented in section 3.3; our main conclusions are drawn in Section 4.

\section{Theoretical Methods}

To provide a description of finite temperature and solvation effects on the the electronic structure of the C343@ NiO interface, we set up a multilevel approach involving both DFT and GW calculations and AIMD simulations. All our static DFT and GW calculations were performed with the Vienna ab initio simulation package (VASP) ${ }^{72-74}$ using the pseudopotentials constructed by the projector augmented wave (PAW) method $^{75}$ and a plane waves basis set. AIMD simulations of the $\mathrm{C} 343 @ \mathrm{NiO}(100)$ interface in explicit water were performed with the $\mathrm{CP} 2 \mathrm{~K}$ code ${ }^{76}$ using 
GTH norm conserving pseudopotentials and MOLOPT-DZVP-SR basis set. To corroborate our GW setup, we first tackle the study of the interface in gas phase and its components, as detailed below. Details about AIMD simulations and GW calculations on the full solvated C343@NiO interface are given in Section 2.2.

\subsection{DFT and GW calculations in vacuo}

Bulk NiO below the Neél temperature $(525 \mathrm{~K})^{77}$ has a type-II antiferromagnetic (AFII) phase that induces a rhombohedral symmetry. All the calculations reported throughout this work have been performed on the AFII phase. Following our previous work, ${ }^{36}$ we modeled the $\mathrm{NiO}$ bulk system starting from the experimental $\mathrm{NiO}$ rock-salt structure, which has a lattice constant of $\sim 4.17 \AA$ (Figure $\mathrm{S} 1$ ).

a)

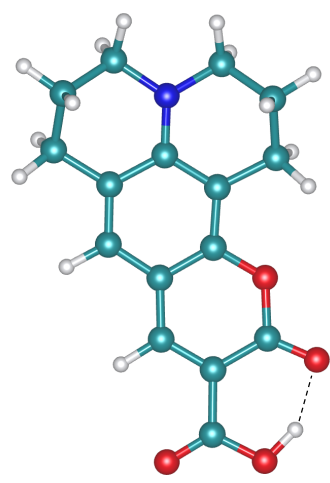

b)

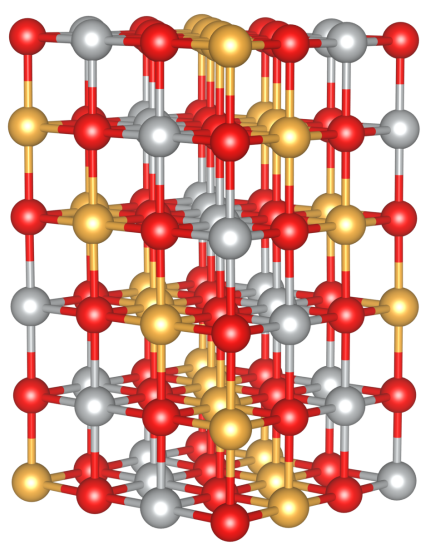

Figure 1: Optimized geometries of the $\mathrm{C} 343$ dye molecule (a) and $\mathrm{NiO}$ surface slab (b). In the latter, oxygen atoms are red, while nichel atoms polarized up/down are gold/silver.

To build the surface slab, we cleaved the bulk structure along the (100) direction and we considered 6 layers in a $(2 \times 2)$ supercell (Figure 1). For the dye, to have direct comparison with the available experiments, we considered here the original C343 molecule, shown in Figure 1, bearing the carboxylic acid as anchoring group. The C343 molecule has been modeled in an orthorhombic cell having ca. $10 \AA$ of vacuum in each direction (Figure S2). 
Ground state optimizations for the C343, NiO (bulk and slab) and C343@ NiO were performed using DFT with the PBE exchange and correlation functional, adding a Hubbard term on the $\mathrm{Ni}(\mathrm{d})$ states. The latter was set equal to $4.6 \mathrm{eV}$, following the value obtained by Cococcioni and de Gironcoli for $\mathrm{NiO}$ with their linear response method. ${ }^{78}$

We used a cutoff of $650 \mathrm{eV}$ for the $\mathrm{NiO}$ systems (bulk, slab and interfaces) and $600 \mathrm{eV}$ for the isolated C343 dye. The Brillouin zone of bulk NiO was sampled using a $8 \times 8 \times 8$ grid of k-points centered at Gamma $(\Gamma)$, whereas for C343, NiO slab and the C343@ NiO interface we considered only the $\Gamma$-point.

The GW calculations have been performed by considering the well known linearized single shot $\mathrm{G}_{0} \mathrm{~W}_{0}$ approximation. ${ }^{79,80}$ Following this approach, the quasi-particle correction associated to the $n^{\text {th }}$ Kohn-Sham (KS) band is obtained as a solution of the following equation: ${ }^{79,80}$

$$
E_{n}^{Q P}=E_{n}^{K S}+Z_{n}\left\langle\phi_{n}^{K S}\left|\Sigma\left(E_{n}^{K S}\right)-V_{x c}\right| \phi_{n}^{K S}\right\rangle
$$

where $Z_{n}$ is known as renormalization factor and corresponds to the derivative of the self energy ( $\Sigma)$ at $E_{n}^{K S}$ :

$$
Z_{n}=\left(1-\operatorname{Re}\left(\left.\frac{\partial\left\langle\phi_{n}^{K S}|\Sigma(\omega)| \phi_{n}^{K S}\right\rangle}{\partial \omega}\right|_{E_{n}^{K S}}\right)\right)^{-1}
$$

The $Z_{n}$ renormalization factor describes the reduction in spectral weight caused by the electronelectron interaction and it represents a measure of the degree of correlation. As can be deduced from Equation 1, the $\mathrm{G}_{0} \mathrm{~W}_{0}$ approximation consists in perturbative corrections of the DFT Kohn-Sham (KS) energies and these corrections have been shown to be highly dependent on the functional adopted in the DFT starting point. ${ }^{81}$ Here, we apply the $\mathrm{G}_{0} \mathrm{~W}_{0}$ correction on the KS energies computed from PBE+U calculations $\left(\mathrm{G}_{0} \mathrm{~W}_{0} @ \mathrm{PBE}+\mathrm{U}\right)$ since, as reported by Scheffler et. al., ${ }^{82}$ this approach delivers energy gaps in good agreement with experiments for most of the transition-metal monoxides, including NiO.

The irreducible polarizability matrix, $\chi_{q}^{0}\left(G, G^{\prime}, \omega\right)$, has been evaluated at the random phase approximation level of theory using a cutoff ( $\mathrm{GW}$ cutoff) of $200 \mathrm{eV}$ for both the isolated C343, the $\mathrm{NiO}$ slab, and the $\mathrm{C} 343 @ \mathrm{NiO}$ interface and $433.3 \mathrm{eV}$ for the $\mathrm{NiO}$ bulk system (see Ref. 74, 83 for further details). For all systems we sampled the polarizability matrix with 100 frequency 
points $(\omega)$. To get converged GW calculations with respect to the number of conduction bands used in the summation over unoccupied bands of the $\chi_{q}^{0}\left(G, G^{\prime}, \omega\right)$, we doubled the considered KS bands until the QP gaps (energies) are converged within an error of 10-20 meV (100-200 meV). This accuracy was achieved by considering 140, 133, 444, 109 unoccupied bands per atom for the $\mathrm{NiO}$ bulk, the $\mathrm{NiO}$ slab, the $\mathrm{C} 343$ and the $\mathrm{C} 343 @ \mathrm{NiO}$ interface, respectively. More details about the convergence of GW calculations are discussed below and in the Supporting Information. Finally, for the NiO slab, the C343@NiO interface, and the isolated C343 molecule, we computed the IP and EA by subtracting from the PBE and GW VBM(HOMO) and CBM(LUMO) the vacuum level that has been evaluated as the electrostatic potential (Hartree plus ionic potential) at the position furthest from the center of the considered system. Having the C343@NiO interface a net dipole moment along the direction perpendicular to the $\mathrm{NiO}$ surface, we applied a dipole corrections ${ }^{84}$ to the total energy and to the electrostatic potential (see the Supporting Information and Figure S10 for further details on the choice of the vacuum level).

\subsection{AIMD, DFT and GW calculations for the C343@ NiO interface in wa- ter}

The AIMD simulations have been carried out using the PBE+U approach considering 500 Ry cutoff for charge density and sampling the Brillouin zone at the $\Gamma$-point. In line with previous studies, ${ }^{85,86}$ to account for van der Waals interactions, we applied Grimme's D3 correction ${ }^{87}$ only to the molecule and to the first surface layer of the $\mathrm{NiO}$ slab, obtaining binding energies similar to the ones reported in the literature for the same system. ${ }^{38}$ The simulation box, having dimensions $8.35 \times 8.35 \times 33.40 \AA^{3}$, consists of the $\mathrm{C} 343 @ \mathrm{NiO}$ interface and 42 water molecules. The time step was set to $1 \mathrm{fs}$ and we impose the deuterium mass for hydrogen atoms. After an initial structural optimization, the MD run was performed in the NVT ensemble by imposing the temperature to $360 \mathrm{~K}$ through the canonical sampling velocity rescaling thermostat. 88

After equilibration (the first 3 ps of the MD simulation), from the production run of $\sim 27$ ps, we extracted 20 configurations equally spaced in time to perform single point $\mathrm{PBE}+\mathrm{U}$ and 
$\mathrm{G}_{0} \mathrm{~W}_{0} @ \mathrm{PBE}+\mathrm{U}$ calculations. The $\mathrm{PBE}+\mathrm{U}$ calculations have been performed with the same cutoff discussed above for the $\mathrm{NiO}$ systems and sampling the Brilloin zone at $\Gamma$ point only. For the GW calculations, we used a cutoff of $200 \mathrm{eV}$ for $\chi_{q}^{0}\left(G, G^{\prime}, \omega\right), 100$ frequency points and we considered a number of KS bands that meet the same ratio between occupied and unoccupied bands needed to converge the calculations on the $\mathrm{C} 343 @ \mathrm{NiO}$ interface in gas phase (77 unoccupied bands per atom).

From these calculations, we evaluate, by means of the projected density of states (PDOS), the energy of the dye $\mathrm{HOMO}\left(E_{\mathrm{HOMO}}\right)$ and the $\mathrm{NiO}$ valence band edge $\left(E_{\mathrm{VBM}}\right)$ and we computed thermal average of the driving force for hole injection $\left(E_{\mathrm{VBM}}-E_{\mathrm{HOMO}}\right)$ and of the energy gaps of the dye and of the $\mathrm{NiO}$ slab.

\section{RESULTS AND DISCUSSION}

\subsection{Isolated systems in vacuo}

\subsubsection{NiO bulk}

In order to validate our computational protocol we start by considering $\mathrm{NiO}$ in its bulk form, which has already been considered in previous ab initio and experimental studies. ${ }^{78,82,89-98}$ The experimental optical gap and the fundamental gap of $\mathrm{NiO}$ reported in the literature span a wide energy range, depending on the method used for the investigation. The optical gap is usually reported in the $3.1-4 \mathrm{eV}$ range where the onset of the absorption is located at about $3 \mathrm{eV}$ and the first well-defined maximum is higher in energy at ca. $4 \mathrm{eV} .{ }^{90-92}$ The fundamental gap measured through photoemission spectroscopy (PES), for occupied states, and bremsstrahlungisochromat spectroscopy (BIS), for unoccupied state, is $4.3 \mathrm{eV},{ }^{93}$ while a value of $4.0 \mathrm{eV}$ was obtained from x-ray absorption (XAS) and emission (XES). ${ }^{89}$ It is well documented in the literature $^{78,94,95}$ that, like for many other transition metal (TM) monoxides, the energy gap of $\mathrm{NiO}$ is dramatically underestimated at PBE level of theory. The addition of an Hubbard term $\mathrm{U}$ to penalize fractional occupations of the $\mathrm{Ni}(\mathrm{d})$ orbitals $(\mathrm{PBE}+\mathrm{U})$, however, delivers values closer to the experimental results.

At the same time, the electronic structure of $\mathrm{NiO}$ is sensitive to the choice of the functional. 


\begin{tabular}{|c|c|c|}
\hline \multirow[t]{2}{*}{ Method } & \multicolumn{2}{|c|}{ Band Gap (eV) } \\
\hline & direct & indirect \\
\hline $\mathrm{PBE}+\mathrm{U}$ & $3.33[0.50 .50 .375]$ & $2.86[\mathrm{~T} \rightarrow 0.50 .250 .25]$ \\
\hline $\mathrm{G}_{0} \mathrm{~W}_{0} @ \mathrm{PBE}+\mathrm{U}$ & $4.27[0.50 .50 .375]$ & $3.80[\mathrm{~T} \rightarrow 0.50 .250 .25]$ \\
\hline \multicolumn{3}{|c|}{ Other theoretical works } \\
\hline $\mathrm{G}_{0} \mathrm{~W}_{0} @ \mathrm{LDA}+\mathrm{U}[82]$ & & 3.75 \\
\hline $\mathrm{G}_{0} \mathrm{~W}_{0} @ \mathrm{HSE03}$ [96] & & 4.70 \\
\hline \multicolumn{3}{|c|}{ Experiment } \\
\hline$E_{g}^{P E S+B I S}[93]$ & & 4.3 \\
\hline$E_{g}^{X A S+X E S}[89]$ & & 4.0 \\
\hline
\end{tabular}

Table 1: Direct and indirect band gaps $(\mathrm{eV})$ of $\mathrm{NiO}$ rocksalt at $\mathrm{PBE}+\mathrm{U}$ and $\mathrm{GW} @ \mathrm{PBE}+\mathrm{U}$ level of theory compared with the experimental gap obtained from PES+BIS and XAS+XES and other theoretical studies. The k-points involved in the band gaps are reported in squared brackets. See Figure S4 for the definition of the high symmetry k-points.

Indeed, as discussed in previous works ${ }^{78,94,95,97,98}$ and shown in Figure S8 in ESI, inclusion of the $\mathrm{U}$ term pushes down in energy the $\mathrm{Ni}(\mathrm{d})$ levels from the top of the valence band and promotes the presence of mixed $\mathrm{Ni}(\mathrm{d})-\mathrm{O}(\mathrm{p})$ states at the valence band maximum $(\mathrm{VBM})$, whereas the conduction band minimum (CBM) is dominated by $\mathrm{Ni}(\mathrm{d})$ states. As shown in Table 1 and evidenced by the band structure in Figure S3 in the Supporting Information, NiO has an indirect band gap. ${ }^{82,95}$ Moreover, in both $\mathrm{PBE}+\mathrm{U}$ and $\mathrm{G}_{0} \mathrm{~W}_{0} @ \mathrm{PBE}+\mathrm{U}$ calculations, the same k-points are involved in both the direct and indirect band gaps. As shown in Table 1, application of the $\mathrm{GW}$ correction on top of $\mathrm{PBE}+\mathrm{U}$ increases the gap by about $0.9 \mathrm{eV}$ (from 2.86 to $3.80 \mathrm{eV}$ ), notably improving the agreement with experiments.

\subsubsection{NiO (100) slab and C343}

GW calculations for the $\mathrm{NiO}$ (100) slab (96 atoms) are extremely expensive and the convergence of the results depends on many variables. In particular, to get converged quasi-particles (QP) energies, and thus QP gaps, for 2D systems, the most critical parameters to consider are: i) the number of k-points; ii) the plane-wave cutoff; iii) the GW cutoff; iv) the number of empty bands and v) the vacuum space along the direction perpendicular to the exposed surface. GW calculations scale quadratically with the number of k-points and, nothwithstanding the choice of considering $\Gamma$-point only represents a non negligible approximation that is only partially mit- 
igated by the size of the slab adopted, the huge computational cost associated with an accurate sampling of the Brillouin zone limited us to consider the Gamma-point approximation. This represents the most significant approximation we made in our approach.
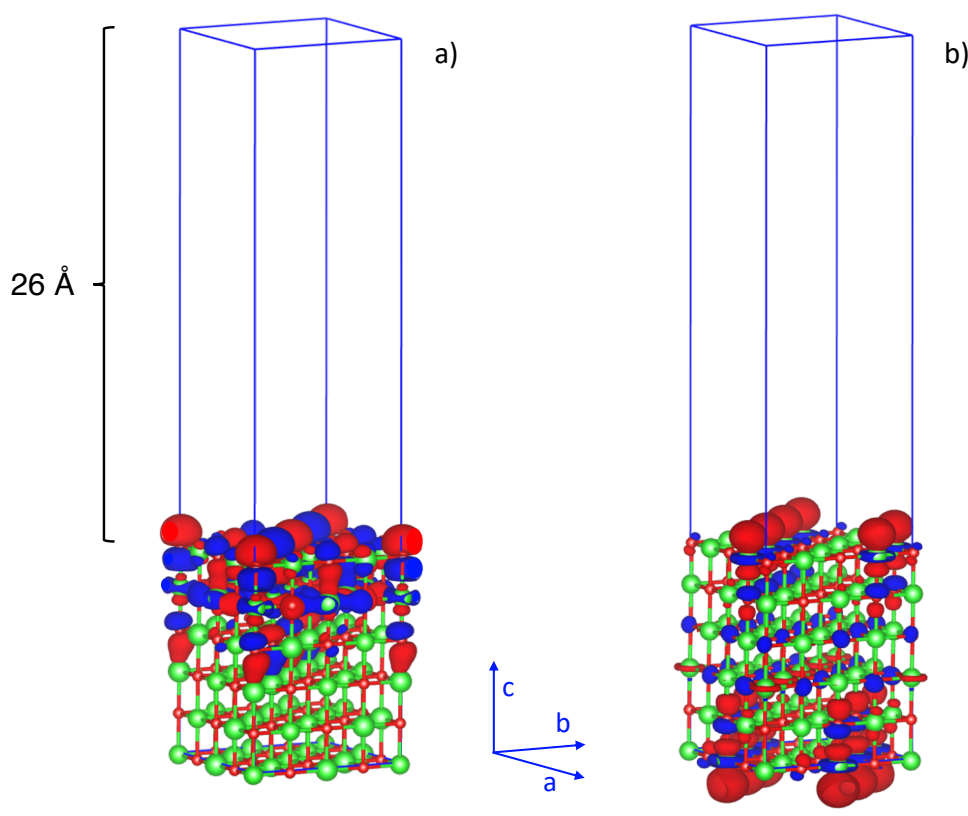

Figure 2: Calculated isosurfaces of the VBM (a) and CBM (b) Kohn-Sham orbitals of the NiO slab system considering $26 \AA$ of vacuum space.

The PBE+U calculations have been found to be weakly affected by the vacuum space due to the semilocal nature of the electrostatic interactions of the GGA functionals; this guarantees also the convergence with respect to the energy cutoff, i.e. there is a negligible effect by increasing the number of plane waves. Conversely, it remarkably influences the convergence of the GW calculations due to the long range nature of the screened Coulomb interactions. ${ }^{99}$

Here we consider the $\Gamma$-point only, which is a reasonable approximation in virtue of the size of the slab adopted, and we analyze the dependence on the vacuum space on GW calculations. Thus, starting from $14 \AA$ of distance between the two exposed surfaces and considering a sufficiently large number of empty bands (1216 empty bands), we increase the vacuum space up to $26 \AA$ where we estimated that convergence was reasonably reached for both the QP energies and QP gap (see Figures S5-S7 in Supporting Information). Then, for definitive GW 
calculations, we converged the number of empty bands following the procedure described in Section 2.1.

Finally, particular attention has to be devoted to the choice of the GW cutoff, which determines the number of $G$ vectors used to build the polarizability matrix and represents the memory bottleneck for GW calculations. The calculations performed for the NiO slab, the isolated C343, the C343@NiO interfaces, which will be reported in the next sections, have been carried out with a different GW cutoff (smaller) compared to the one used for the $\mathrm{NiO}$ bulk system, as discussed in Section 2. However, as shown in Table A in the Supporting Information, the use of the same cutoff as the one used for the such systems is giving only negligible difference in the calculated direct and indirect energy gap for the $\mathrm{NiO}$ bulk system, hence confirming the accuracy of our set up.

The converged PBE+U and GW gaps are reported in Table 2, where one can notice that for the slab these values are lower than the ones obtained for the bulk in Table 1. In fact, even though the PDOS of the NiO slab has a nature similar to the one of the bulk (Figure S8b in Supporting Information), in the slab the band edges, due to the under-coordination of the atoms on the two surface layers, ${ }^{100-102}$ are dominated by surface states as shown in Figure 2, where the isodensity plots of the VBM (a) and CBM (b) are displayed.

\begin{tabular}{l|ccc}
\hline \hline Method & $E_{g}$ & IP & EA \\
\hline \multicolumn{4}{|c}{$N i O(100)$} \\
\hline PBE+U & 2.57 & 5.49 & 2.92 \\
$\mathrm{G}_{0} \mathrm{~W}_{0} @ \mathrm{PBE}+\mathrm{U}$ & 3.55 & 5.66 & 2.11 \\
Exp. & $4.0{ }^{89} 4.3^{93}$ & $\sim 5^{103}$ & $\sim 1.8^{103}$ \\
\hline \hline \multicolumn{4}{|c}{$C 343$} \\
$\mathrm{PBE}$ & 2.23 & 5.27 & 3.04 \\
$\mathrm{G}_{0} \mathrm{~W}_{0} @ \mathrm{PBE}$ & 5.66 & 6.82 & 1.16 \\
evGW@LDA & 6.61 & 7.21 & 0.60 \\
\hline \hline
\end{tabular}

Table 2: Energy gap, ionization potential (IP) and electron affinity (EA) in eV for the $\mathrm{NiO}$ (100) slab and the $\mathrm{C} 343$ dye computed using $\mathrm{PBE}+\mathrm{U}$ and $\mathrm{G}_{0} \mathrm{~W}_{0} @ \mathrm{PBE}+\mathrm{U}$

We also computed the ionization potential (IP) and the electron affinity (EA), see Table 2, of the slab as the negative of the VBM and CBM KS eigenvalues and QP energies relative to vacuum level (r.t.v.). ${ }^{104}$ The experimental value of the VBM usually reported is $-5 \mathrm{eV}^{103}$ r.t.v., 
although, as for the band gap, it depends on the adopted measurement technique. Indeed, this varies from the $-4.8 \mathrm{eV}(\mathrm{PES})^{105}$ to $-5.0 \mathrm{eV}$ (electrochemical measurement) ${ }^{103,106}$ to -5.8 $\mathrm{eV}$ (thermionic emission). ${ }^{107}$ Measured EAs are not available for $\mathrm{NiO}$, so the experimental EA values are obtained by subtracting the experimental band gap from the experimental IP. For the sake of simplicity, we refer to the value of $-1.8 \mathrm{eV},{ }^{103}$ since it is the one reported most frequently in the literature.

The GW corrections (Table 2) improve the overall agreement with experiments, delivering an $E_{g}$ of $3.55 \mathrm{eV}$ and IP and EA values of 5.66 and $2.11 \mathrm{eV}$, respectively. In particular, as reported for a large set of semiconductors, ${ }^{108} \mathrm{GW}$ reduces the over-stabilization of the EA obtained at DFT level. On the other hand, the experimental IP is slightly better reproduced from DFT calculations, being $0.17 \mathrm{eV}$ smaller than that predicted from $\mathrm{GW}$ calculations. However, we should emphasize that the spread in the measurements reported for both band gap and IP as well as the possible difference between our slab model and $\mathrm{NiO}$ systems considered in refs. 89,93,103 makes an unbiased comparison rather difficult.

Then, for the $\mathrm{C} 343$ molecule, from the PBE and the $\mathrm{G}_{0} \mathrm{~W}_{0} @ \mathrm{PBE}$ calculations we computed the values for IP, EA and $E_{g}$ by considering the HOMO and LUMO values with respect to the electrostatic potential along the $b$ direction (Figure S2), where the dipole moment has negligible magnitude. As far as we are aware, there are no reported experimental IP and EA for C343 in gas phase, so here we compare our results with those reported by Blase and co-workers ${ }^{62}$ obtained by partially self consistent GW (evGW) on the top of LDA calculation. Values in Table 2 show an overall good agreement between our $\mathrm{G}_{0} \mathrm{~W}_{0}$ IP and EA values and the more accurate evGW ones. However, the two approaches predict band gaps that differ by about $1 \mathrm{eV}$, highlighting the high contribution from iterating $G$ and $W$ in $\mathrm{GW}$ calculations on isolated molecules. In both cases the energy gap is sizeably larger than the C343 optical absorption, that, in apolar solvent (ciclohexane) shows an absorption maxima at ca. $3 \mathrm{eV},{ }^{109}$ thus evidencing a huge electron-hole binding energy, as it has been already reported for isolate conjugated molecules in gas phase. ${ }^{110}$

In this section we validated our methodology considering the standalone elements of the C343@NiO interface, finding an overall good agreement with the available experimental data and with other theoretical investigations. In the next section we will address the study of the 
C343@NiO interface.

\subsection{C343@ NiO Interface in vacuo}

Following previous findings, ${ }^{38}$ we considered the $\mathrm{C} 343$ dye anchored in a bidentate fashion (Figure 3a) to the (100) $\mathrm{NiO}$ surface slab. In order to minimize spurious interactions between periodic images, we imposed a vacuum space of $26 \AA$ between the two surfaces of NiO.

a)

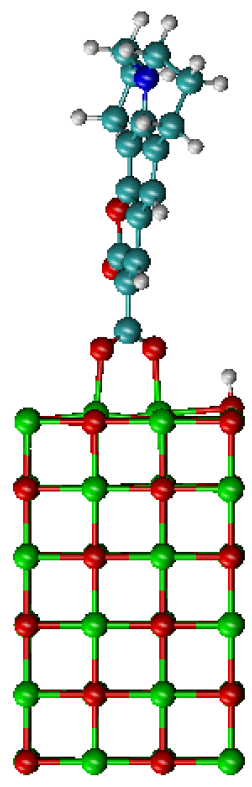

b)

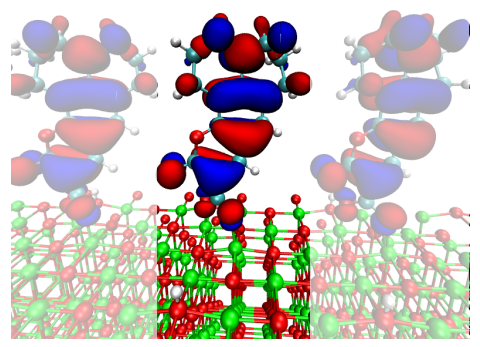

c)

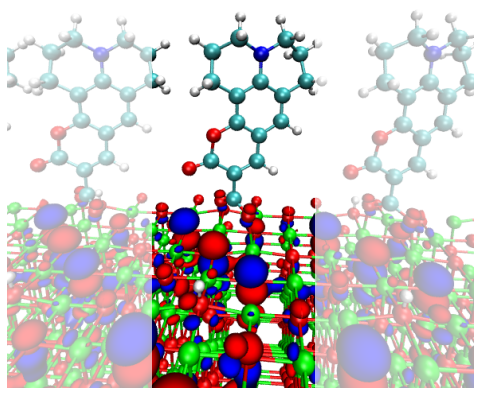

Figure 3: Optimized C343@NiO system in gas phase a) and isodensity plots of the dye HOMO (b) and NiO VBM (c) Kohn-Sham orbitals.

As shown by the isodensity plots in panels b and c of Figure 3, dye HOMO and the $\mathrm{NiO}$ VBM present a negligible electronic coupling, ${ }^{36}$ since the orbitals are strongly localized on the molecule and on the slab, respectively. Thus, once identified the C343 HOMO/LUMO and the VBM/CBM bands by means of the PDOS (Figure S9), the energy alignment between the frontier KS orbitals, the energy gaps and driving forces for hole injection can be straightforwardly obtained (Figure 4) and compared with the ones given by the calculations on the isolated dye and semiconductor slab (Table 2). 
Let us first discuss the results obtained at PBE+U level of theory (grey lines in Figure 4). The NiO gap calculated when the dye is grafted on the surface turns out to be $\sim 0.1 \mathrm{eV}$ smaller than the one obtained for the isolated slab, due to a larger up-shift of the VBM with respect to the CBM, possibly caused by structural modifications and charge transfer/electrostatic effects following the deprotonated dye anchoring. On the other hand, the calculated HOMO/LUMO gap of the dye increases (by $\sim 0.2 \mathrm{eV}$ ) when it is anchored on the slab, due to a sizeable destabilization (by $\sim 1 \mathrm{eV}$ ) of the LUMO.

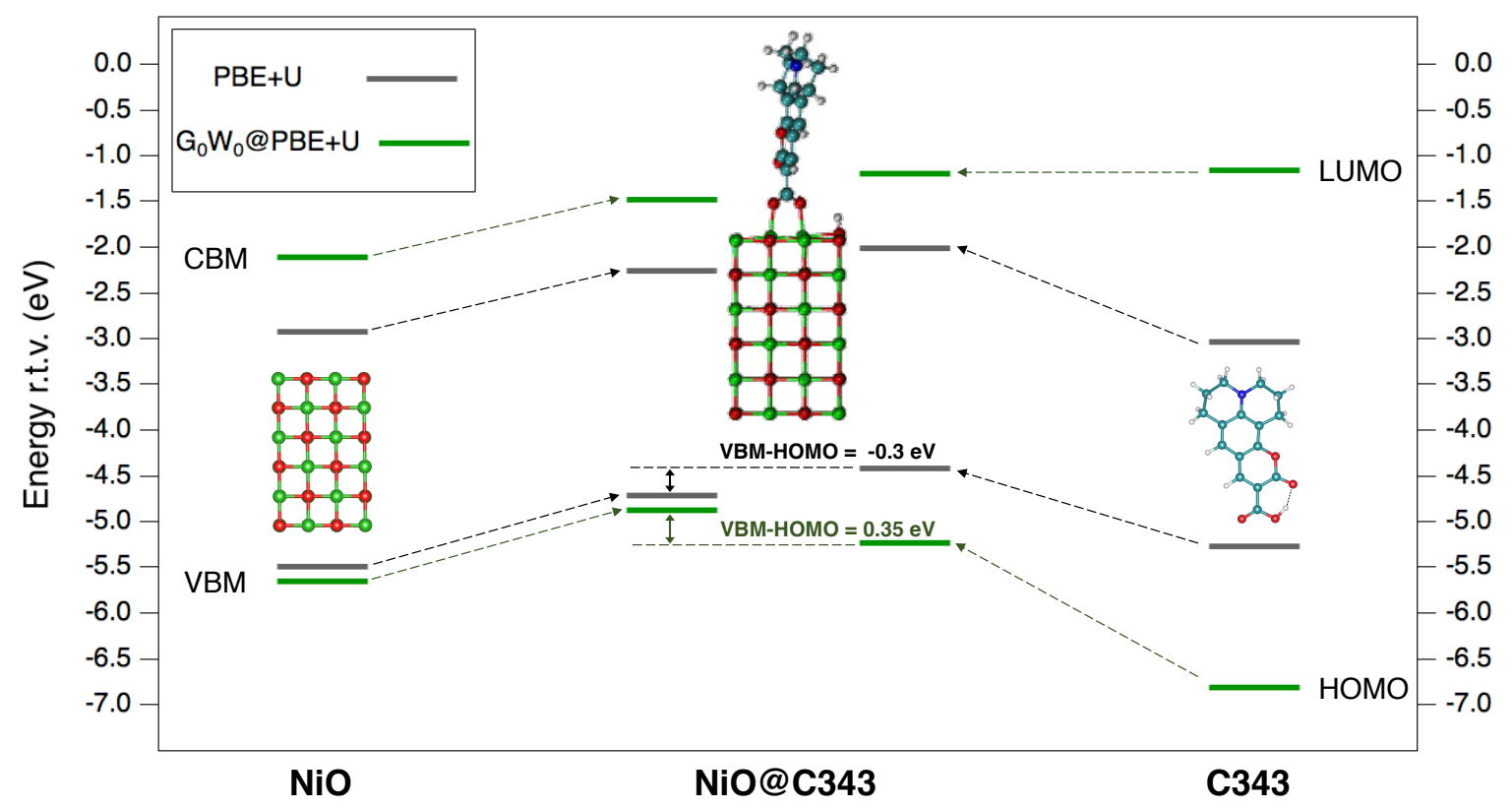

Figure 4: $\mathrm{PBE}+\mathrm{U}$ (grey lines) and $\mathrm{G}_{0} \mathrm{~W}_{0} @ \mathrm{PBE}+\mathrm{U}$ (green lines) energy levels with respect to the vacuum of the $\mathrm{C} 343 \mathrm{HOMO} / \mathrm{LUMO}$ and $\mathrm{NiO} \mathrm{VBM} / \mathrm{CBM}$ as computed for the isolated systems (data in Table 2) and for the C343@NiO interface model. The driving forces for the hole injection from the dye to the semiconductor (VBMHOMO) are reported. The dashed arrows are a guide for the eye to indicate the passage from isolated systems (left: $\mathrm{NiO}$, right: $\mathrm{C} 343$ ) to the $\mathrm{C} 343 @ \mathrm{NiO}$ interface (center).

This effect is due to geometrical distortion of the C343 structure driven by the deprotonation and bonds with the $\mathrm{Ni}$ atoms of the $\mathrm{NiO}$ surface and by the interactions between periodic images along the $\mathrm{a}$ and $\mathrm{b}$ directions. ${ }^{56}$ However, these interactions are not to be regarded as spurious because the surface dye concentration adopted in our model of the interface $(\sim 1$ molecule/ 0.70 $\mathrm{nm}^{2}$ ) is in line with the typical experimental surface coverage employed in DSSCs. ${ }^{111,112}$ 
As far as the VBM-HOMO difference is concerned, a negative driving force of $-0.3 \mathrm{eV}$ is predicted for the interface in gas phase. By taking the HOMO and VBM values calculated for the isolated systems, this difference is mitigated and amounts to $-0.22 \mathrm{eV}$, though it is still unfavourable for holes transfer.

The $\mathrm{G}_{0} \mathrm{~W}_{0}$ correction (green lines in Figure 4), as already discussed for the standalone components, brings about a sizeable increase in the energy gaps and relevant differences in the electronic structure (Figure S9, bottom panel). The NiO gap (that now turns out to be the gap of the interface system) results to be, again, close to the gap calculated on the isolated slab and the small decrease $(\sim 0.15 \mathrm{eV})$ is mainly due to the stabilization of the CBM. On the other hand, the QP dye gap is significantly reduced (by $\sim 1.6 \mathrm{eV}$ ) compared to the one obtained from the isolated molecule and this effect is almost entirely ascribable to the up-shift of the HOMO. This marked gap reduction predicted from the GW calculation has already been reported for comparable interface systems ${ }^{56,113}$ and depends on image charge effects that are not correctly described at the bare DFT level, where the gap is usually unchanged when going from the isolated molecule to the molecule at the interface. ${ }^{113-115}$ Moreover, these effects reduce also the exciton binding energy, as shown by Garcia-Lastra et. al. for molecule physisorbed on metallic surfaces, ${ }^{113,115}$ and produce a QP gap much more similar to the experimental optical gap reported in Section 3.1.

The most striking result coming from the $\mathrm{G}_{0} \mathrm{~W}_{0}$ correction, however, concerns the relative alignment between the $\mathrm{C} 343 \mathrm{HOMO}$ and the VBM of the adsorbed system. At the GW level the sign of the driving force is inverted with respect to the DFT results, giving a slightly positive value of $0.35 \mathrm{eV}$. This is the result of a differential perturbative correction on the dye and $\mathrm{NiO}$ states, coming from the different nature of the QPs. This difference can be quantified by the $\mathrm{Z}$ renormalization factor, which is in the order of 0.8 for the $\mathrm{C} 343$ states, while it ranges between 0.6-0.7 for the surface slab states. ${ }^{116,117}$

It is worth stressing that this change in the dye and semiconductor relative energy levels alignment cannot be attained using hybrid functionals, since they only produce an opening of the energy gap of the interface system, without altering the alignment between the molecule and semiconductor KS eigenvalues, thus delivering very similar driving forces. ${ }^{36}$ 
To sum up, in this section we have shown that GW calculations on the model dye/semiconductor system in gas phase can recover the correct interfacial energetics (a positive driving force), which is missed at DFT+U level. As we shall discuss in the next section, inclusion of the explicit solvation effects will further refine our picture, allowing for a quantitative comparison with experimental estimations.

\subsection{C343@NiO interface in water}

Dye sensitizers bearing carboxylic anchoring groups are often prone to undergo desorption from metal oxides surfaces in water environment ${ }^{118,119}$ and the use of phosphonic acid group is, usually, recommended to guarantee a better long-term stability of the cell. Water chemisorption on $\mathrm{NiO}$ and on other oxide SCs is indeed a well-known phenomenon, reported by both experimental and theoretical investigations ${ }^{120-122}$ and the competitive adsorption between water molecules and carboxylates at the metal oxide interface is the reason for the observed dye desorption. ${ }^{118,123}$

The first point to assess is, therefore, the stability of C343 on the NiO surface. Along the 30 ps trajectory the dye did not desorb, although we observe after around 7 ps a dynamic interconversion between the bidentate and monodentate anchoring, driven by thermal fluctuations and by the interaction with the surrounding water molecules, as shown by the distance fluctuations of one of the two Ni-O bonds (green line) in the top panel of Figure 5.

Along the MD run we observed the dissociative adsorption $\left(\mathrm{H}^{+}\right.$and $\left.\mathrm{OH}^{-}\right)$of two water molecules, one on each face of the $\mathrm{NiO}$ slab. As evidenced by the interatomic pair distribution function $\left(\mathrm{g}_{\alpha \beta}(r)\right)$ between the hydrogen atoms of the water molecules and the $\mathrm{NiO}$ oxygen atoms (Figure S11), within the first $\sim 3$ ps one of the two water molecules is already chemisorbed, giving rise to the peak at $1 \AA$; the second water molecule, the one at the $\mathrm{C} 343 / \mathrm{NiO}$ interface, is adsorbed after 6-7 ps, as attested by the increase in the intensity of the peak of the $\mathrm{g}_{\alpha \beta}(r)$ in the time interval 3-7 ps. The intense narrow peak at $1 \AA$ presents in the $\mathrm{g}_{\alpha \beta}(r)$ computed over the last part of the dynamics ( $7 \mathrm{ps}<\mathrm{t} \leq 30 \mathrm{ps}$ ) indicates that the two water molecules remain stably chemisorbed on the $\mathrm{NiO}$ surfaces and thus the adsorbed oxydril close to the molecule can interact with the anchoring unit of C343 (bottom panel of Figure 5), induc- 

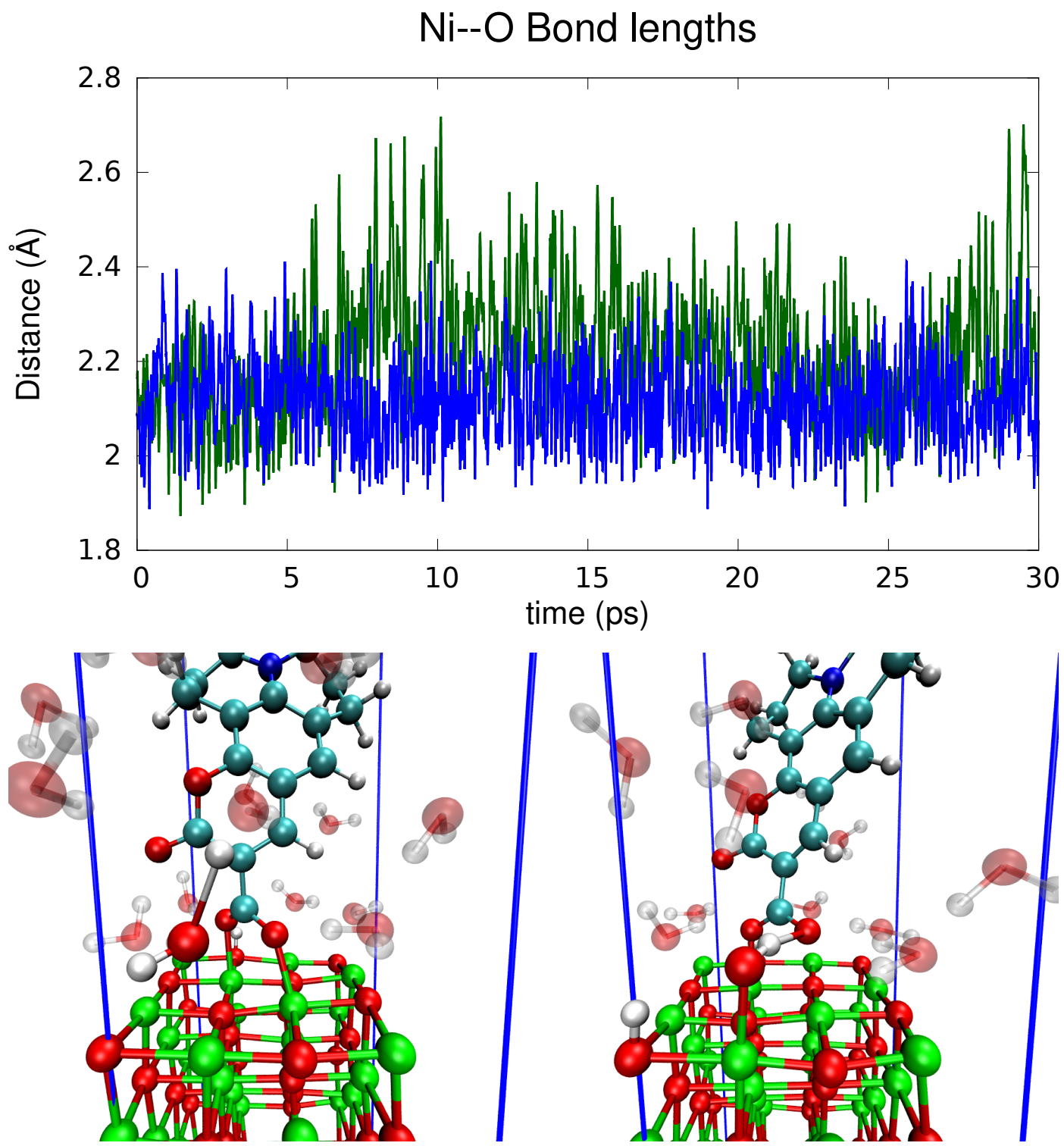

Figure 5: Top: Ni-O(C343) bond distances computed along the 30 ps trajectory; bottom: two snapshots extracted at $0.5 \mathrm{ps}$ (left) and $7 \mathrm{ps}$ (right) showing the water chemisorption at the C343@NiO interface and the interaction between the adsorbed $\mathrm{OH}^{-}$and the $\mathrm{C} 343$ anchoring group.

ing the elongation of the O-Ni bond discussed above (top panel of Figure 5).

In order to analyze in detail the role of the solvent on the electronic properties of $\mathrm{C} 343 @ \mathrm{NiO}$, we considered the interface structure after the 3 ps of equilibration, that is the first frame of the MD production trajectory. By focusing on one snapshot, we can, indeed, try to disentangle the 


\begin{tabular}{l|ccc}
\hline \hline Method & $E_{g}^{D Y E}$ & $E_{g}^{N i O}$ & VBM-HOMO \\
\hline \multicolumn{4}{c}{ solvated } \\
\hline PBE+U & 2.10 & 2.39 & 0.37 \\
$\mathrm{G}_{0} \mathrm{~W}_{0} @ \mathrm{PBE}+\mathrm{U}$ & 3.38 & 3.46 & 0.70 \\
\hline \multicolumn{4}{c}{ Experiment } \\
\hline Exp. & $\sim 3.0^{44}$ \\
\hline \multicolumn{4}{c}{ "vacuum” } \\
\hline $\mathrm{PBE}+\mathrm{U}$ & 2.32 & 1.76 \\
$\mathrm{G}_{0} \mathrm{~W}_{0} @ \mathrm{PBE}+\mathrm{U}$ & 3.77 & 2.89 & 0.27 \\
\hline \hline
\end{tabular}

Table 3: Calculated PBE+U and $\mathrm{G}_{0} \mathrm{~W}_{0} @ \mathrm{PBE}+\mathrm{U}$ dye gap (LUMO-HOMO), NiO gap (CBMVBM) and driving force (VBM-HOMO) for the initial snapshot of the MD production run (after 3 ps of equilibration). The values computed on the explicitly solvated NiO/C343 interface (solvated) are compared with the ones calculated by removing all the water molecules ("vacuum"). Experimental available data are also reported. All values are in $\mathrm{eV}$.

mechanical embedding effects from the electrostatic and polarization ones, by comparing the energy levels alignment of the full solvated dye/NiO interface extracted from the MD snapshot and the one obtained on the same snapshot by removing the water molecules (hereafter referred as "vacuum"). The $\mathrm{PBE}+\mathrm{U}$ and $\mathrm{G}_{0} \mathrm{~W}_{0} @ \mathrm{PBE}+\mathrm{U}$ energy gaps and driving forces are listed in Table 3.

Also in water, the dye levels and the $\mathrm{NiO}$ bands are essentially electronically decoupled (see the isodensity plots in Figure S12 in Supporting Information), thus the energy gaps and the driving force can be straightforwardly obtained by the dye HOMO/LUMO and NiO VBM/CBM peaks in the PDOSs. As expected on the basis of our previous findings, ${ }^{36}$ explicit solvation has a major effect on the interfacial energetics (Table 3), by relatively shifting the levels of the dye and of the $\mathrm{NiO}$ slab in the direction of increasing the driving force for the hole injection. As is apparent in Table 3, indeed, already at $\mathrm{PBE}+\mathrm{U}$ level, the driving force is positive, passing from $-0.3 \mathrm{eV}$, for the optimized interface in vacuo (top panel of Figure 4), to $+0.37 \mathrm{eV}$. A larger driving force with respect to the one calculated in vacuo (bottom panel of Figure 4) is also obtained at GW level of theory, where the calculated VBM-HOMO difference is $0.7 \mathrm{eV}$, quantitatively agreeing with the experimental estimation of the driving force. ${ }^{44,45}$

We can quantify the effect of the geometrical distortions induced by the presence of the solvent, by looking at the data obtained for the "vacuum" calculation on the same frame (bottom 
of Table 3) after removing all water molecules and applying the dipole corrections in the middle of the vacuum space to determine the reference vacuum level. The resulting driving forces and energy gaps are rather different from the ones calculated from the optimized structure in vacuo (Figure 4), with an overall reduction of the gaps, and a driving force going from -0.3 and 0.35 $\mathrm{eV}$ to 0.27 and $0.48 \mathrm{eV}$ at $\mathrm{PBE}+\mathrm{U}$ and $\mathrm{G}_{0} \mathrm{~W}_{0} @ \mathrm{PBE}+\mathrm{U}$ level, respectively. If we compare these driving forces with those discussed above and calculated on the explicitly solvated interface, interestingly we disclose that the mechanical effect dominates over the electrostatic and polarization effects, already accounting for about $73 \%$ and $69 \%$ of the total driving force calculated by $\mathrm{DFT}+\mathrm{U}$ and GW, respectively.

By looking at the absolute values of the calculated KS and QP energies (Table B in Supporting Information) and comparing them with those reported in Figure 4, we can trace back the changes of the $\mathrm{NiO}$ gap mainly to the down-shift of the CBM (about $0.4 \mathrm{eV}$ ), that, being localized on the surface (see Figure 3c) are strongly affected by the distortion of the first layer of the slab caused by the interaction with the adsorbed water molecules. The root mean square deviations (RMSD) for the atoms belonging to the first layer, calculated with respect to the optimized interface in vacuo, is indeed higher $(0.16 \AA)$ compared to the one computed for the second $(0.11 \AA)$ and the third layer $(0.10 \AA)$. The larger fluctuations of the first surface layer with respect to inner layers is displayed in Figure S13, where the RMSDs of the three uppermost layers calculated along the MD run are plotted.

Having rationalized the effect of the solvent at the dye/ $\mathrm{NiO}$ interface on the initial configuration (i.e., the snapshot extracted after 3 ps), we can now discuss the energy level alignment along the whole trajectory. To do so we considered 20 snapshots, equally spaced in time, extracted from the MD production run, and we performed PBE+U and $\mathrm{G}_{0} \mathrm{~W}_{0} @ \mathrm{PBE}+\mathrm{U}$ calculations on the full solvated interface model. The time evolution of the calculated driving force and energy gaps are plotted in Figure 6, while the values with the corresponding standard deviation and standard error are listed in Table $\mathrm{C}$ in Supporting Information along with those obtained considering only 10 snapshots. The small differences found between the two samplings reveal that the convergence of the calculated properties has been already reasonably achieved with 20 snapshots. It is meaningful to compare the thermal-averaged gaps and driving forces with those 

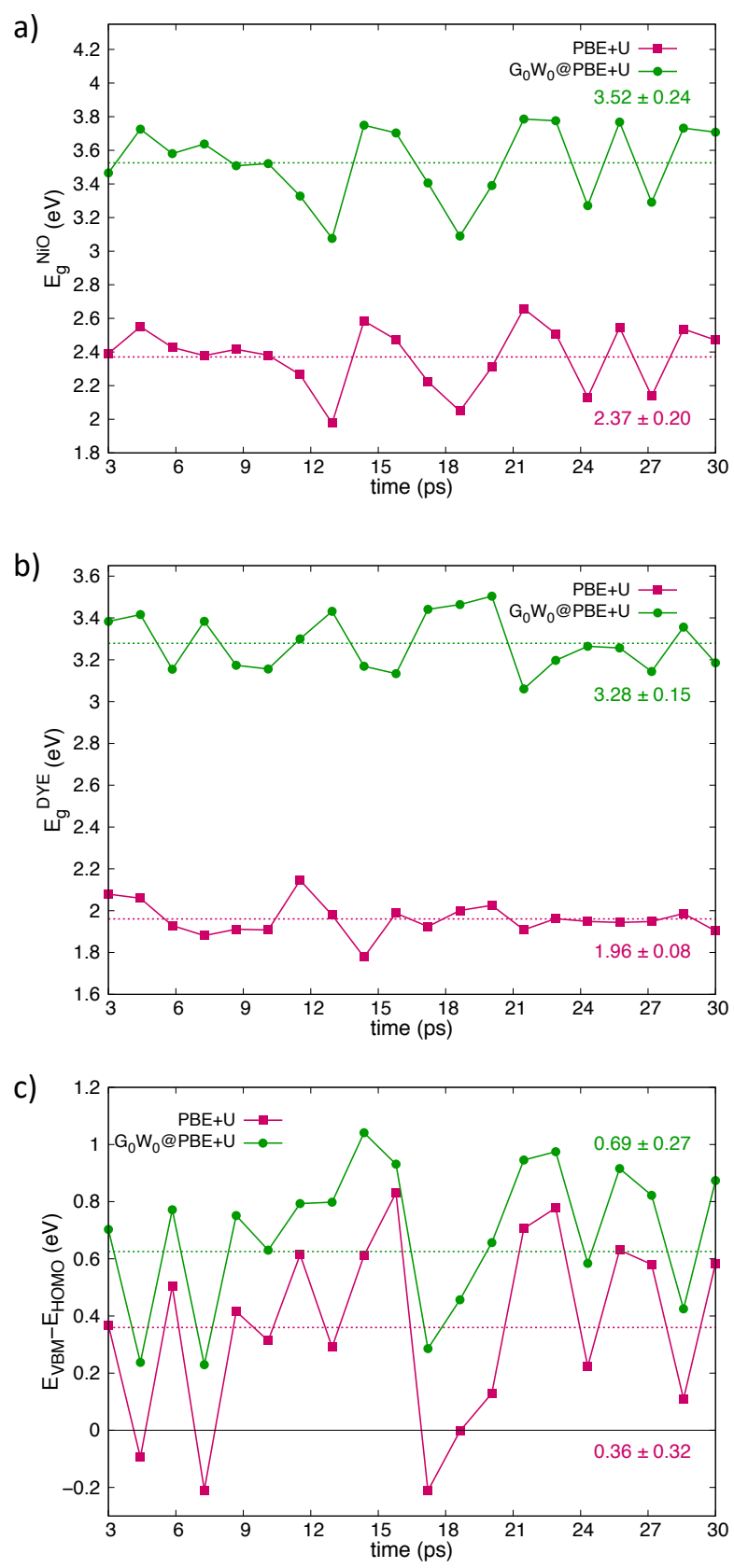

Figure 6: Calculated NiO gap (a), Dye gap (b) and difference between the VBM of the NiO and the HOMO of the Dye (c) along the MD production run in eV considering both the $\mathrm{PBE}+\mathrm{U}$ and the $\mathrm{G}_{0} \mathrm{~W}_{0} @ \mathrm{PBE}+\mathrm{U}$ methods.

of the static picture at the beginning of the MD simulations. Along the dynamics, we observe an overall band gap shrinkage for both dye and $\mathrm{NiO}$, with larger fluctuations at GW level of theory. Interestingly, as shown by panels a) and b) in Figure 6, while for the $\mathrm{NiO}$ slab the gap 
evolution at GW level approximately follows the $\mathrm{PBE}+\mathrm{U}$ one, the correlation between the DFT and the GW corrected values appears less evident for the dye, where the GW results are more scattered.

The QP NiO band gap calculated over the MD trajectory is $3.52 \pm 0.24 \mathrm{eV}$, in better agreement with the experiments. ${ }^{45,124}$ The same applies for the dye: the QP gap is slightly smaller compared to the static picture discussed above $(3.28 \pm 0.15)$, therefore it remains smaller than the gap calculated in the gas phase, evidencing a bathochromic shift in agreement with experimental evidence. ${ }^{109}$ The most interesting result, however, concerns the VBM-HOMO differences (Figure 6c). We find that the driving force for hole injection is always significantly larger at GW level (average value of $0.69 \pm 0.27 \mathrm{eV}$ ) compared to the values obtained with $\mathrm{PBE}+\mathrm{U}$ (average value of $0.36 \pm 0.32 \mathrm{eV}$ ), and in quantitative agreement with the experimental estimation in the range 0.6-1.0 eV. More in detail, the $\mathrm{GW}$ driving force is always positive, i.e. favourable for hole injection, and oscillates between $\simeq 1 \mathrm{eV}$ and $\simeq 0.1 \mathrm{eV}$. The $\mathrm{GW}$ correction is also very different from one snapshot to the other, going from 0.1 to $0.6 \mathrm{eV}$. It is worth noting that in Ref.36 the driving forces obtained by using the hybrid HSE06 functional were in the same range of the $\mathrm{PBE}+\mathrm{U}$ ones, differing by less than $0.1 \mathrm{eV}$, due to a similar effect on both the $\mathrm{VB}$ of $\mathrm{NiO}$ and on the $\mathrm{HOMO}$ of the dye. ${ }^{49}$ It is important to stress here that accessing the energetics of dye/solvent/SC interfaces in water solution by XPS measurements is extremely challenging, ${ }^{125,126}$ as attested by the fact that no photoelectron spectroscopy measurements on in situ or ex situ the dye-sensitized $\mathrm{NiO}$ interface are available and that the experimental driving force is estimated using the red-ox potentials of isolated C343 and NiO. One should, however, keep in mind that this can be a rather crude approximation, justifying the rather large range of the experimental driving force. Looking at the more extensively investigated dye/ $/ \mathrm{TiO}_{2}$ interface, indeed, large differences on the energy levels of the molecules when anchored on the substrate were reported ${ }^{127}$ and, moreover, the relative alignment between the dye HOMO and the semiconductor VBM may change significantly in the presence of the solvent. ${ }^{126}$ In this context, having a computational protocol, benchmarked against the data available for the isolated dye and semiconductor and capable to deliver an accurate estimation of the structure and energetics of the interface, may represent an effective tool to get precise information on the 
thermodynamics of the hole injection process and consequently to tune the chemical structure and the optoelectronic properties of the dye sensitizer.

\section{Conclusions}

By considering a simplified, albeit realistic, model of a dye-sensitized $\mathrm{NiO}$ interface (C343@ $\mathrm{NiO}(100)$ ), we have shown that GW calculations, coupled to AIMD simulations in explicit solvent, are capable to deliver, in quantitative agreement with experiments, the interfacial energy-level alignment required for the functioning of p-type photocathodes.

GW method accounts for the anisotropic nature of the screening in interfacial systems and hence, in the case of $\mathrm{C} 343$ sensitized $\mathrm{NiO}$ interface, we observe a different perturbative correction for the dye and the substrate states. As a result, the energy-level alignment is different compared to the DFT one, as evidenced by the calculations on the $\mathrm{C} 343 @ \mathrm{NiO}$ interface in vacuo, wherein the driving force changes sign upon GW corrections, thus favouring a beneficial VBM-HOMO alignment for hole transfer. This result cannot be achieved using standard hybrid functionals, as they act similarly on both dye levels and $\mathrm{NiO}$ levels and therefore they do not significantly alter the energy level alignment. It is worth stressing that the alignment is largely affected by electrostatic and mechanical effects induced by the dye anchoring and by the solvent (water) layer adsorbed to the surface, however, the accuracy of the employed electronic structure method is, on the other hand, crucial to get a quantitative estimation of the energy difference between the NiO VBM and the dye HOMO (i.e. the driving force for the hole injection process).

Dynamical effects, image charge effects and solvent polarization effects plays a key role in dictating the properties of the $\mathrm{C} 343 @ \mathrm{NiO}$ interface, by strongly affecting the dye and $\mathrm{NiO}$ energy gaps, and cannot be neglected when estimating from first principles the optoelectronic properties of these photoactive hybrid interfaces. This contribution represents the first example of the feasibility of accurate large scale GW calculations on realistic models of dye-sensitized metal oxide interfaces, including solvent molecules and finite temperature effects. 


\section{Supporting Information}

Optimized structures of $\mathrm{C} 343, \mathrm{NiO}$, and the C343@NiO interface both in vacuo and in water. First Brillouin zone and band structure of NiO. DOS and PDOS for the NiO bulk, NiO slab and the C343@NiO interface. Convergence tests on GW calculations on the $\mathrm{NiO}$ bulk and the NiO slab. Details on the band edge alignement. Analysis of the MD trajectories: atomic pair correlation functions calculated between the oxygen atoms of the $\mathrm{NiO}$ surfaces and the hydrogen atoms belonging to the water molecules; RMSD of the first three uppermost layers of the NiO. Isosurfaces of the HOMO, LUMO, VBM, and CBM Kohn-Sham orbitals of the Interface system in water solution. GW Energy gaps and VBM-HOMO difference calculated along the MD run of the $\mathrm{C} 343 @ \mathrm{NiO}$ in explicit water.

\section{Acknowledgements}

The work has been performed under the Project HPC-EUROPA3 (INFRAIA-2016-1-730897), with the support of the EC Research Innovation Action under the H2020 Programme. M.P and A.S. thank the financial support from the ANR JCJC HELIOSH2 (ANR-17-CE05-0007-01) grant and the COMETE project (COnception in silico des Matériaux pour l'EnvironnemenT et l'Energie) co-funded by the European Union under the program 'FEDER-FSE Lorraine et Massif des Vosges 2014-2020. HPC resources from GENCI-CCRT/CINES (Grants 2018A0010810139) and from the LPCT local computing clusters are also acknowledged. 


\section{References}

[1] O’Regan, B.; Grätzel, M. A low-cost, high-efficiency solar cell based on dye-sensitized colloidal TiO2 films Nature 1991, 353, 737-740.

[2] Lee, C.-P.; Li, C.-T.; Ho, K.-C. Use of organic materials in dye-sensitized solar cells Mater. Today 2017, 20, 267 - 283.

[3] Nikolaou, V.; Charisiadis, A.; Charalambidis, G.; Coutsolelos, A. G.; Odobel, F. Recent advances and insights in dye-sensitized $\mathrm{NiO}$ photocathodes for photovoltaic devices $J$. Mater. Chem. A 2017, 5, 21077-21113.

[4] He, J.; Lindström, H.; Hagfeldt, A.; Lindquist, S.-E. Dye-Sensitized Nanostructured pType Nickel Oxide Film as a Photocathode for a Solar Cell J. Phys. Chem. B 1999, 103, 8940-8943.

[5] Gong, J.; Liang, J.; Sumathy, K. Review on dye-sensitized solar cells (DSSCs): Fundamental concepts and novel materials Renew. Sustain. Energy Rev. 2012, 16, 5848 5860 .

[6] Pastore, M.; De Angelis, F. Modeling materials and processes in dye-sensitized solar cells: understanding the mechanism, improving the efficiency Top. Curr. Chem. 2014, $352,151-236$.

[7] Kakiage, K.; Aoyama, Y.; Yano, T.; Oya, K.; Fujisawa, J.-i.; Hanaya, M. Highly-efficient dye-sensitized solar cells with collaborative sensitization by silyl-anchor and carboxyanchor dyes Chem. Commun. 2015, 51, 15894-15897.

[8] Renaud, A.; Cario, L.; Pellegrin, Y.; Blart, E.; Boujtita, M.; Odobel, F.; Jobic, S. The first dye-sensitized solar cell with p-type LaOCuS nanoparticles as a photocathode $R S C A d v$. 2015, 5, 60148-60151. 
[9] Benazzi, E.; Mallows, J.; Summers, G. H.; Black, F. A.; Gibson, E. A. Developing photocathode materials for p-type dye-sensitized solar cells J. Mater. Chem. C 2019, 7, 10409-10445.

[10] Jiang, T.; Bujoli-Doeuff, M.; Farré, Y.; Pellegrin, Y.; Gautron, E.; Boujtita, M.; Cario, L.; Jobic, S.; Odobel, F. CuO nanomaterials for p-type dye-sensitized solar cells RSC Adv. 2016, 6, 112765-112770.

[11] Zhang, X.; Peng, T.; Song, S. Recent advances in dye-sensitized semiconductor systems for photocatalytic hydrogen production J. Mater. Chem. A 2016, 4, 2365-2402.

[12] Warnan, J.; Gardner, J.; Le Pleux, L.; Petersson, J.; Pellegrin, Y.; Blart, E.; Hammarström, L.; Odobel, F. Multichromophoric Sensitizers Based on Squaraine for NiO Based Dye-Sensitized Solar Cells J. Phys. Chem. C 2014, 118, 103-113.

[13] Sherman, B. D.; Sheridan, M. V.; Wee, K.-R.; Marquard, S. L.; Wang, D.; Alibabaei, L.; Ashford, D. L.; Meyer, T. J. A Dye-Sensitized Photoelectrochemical Tandem Cell for Light Driven Hydrogen Production from Water J. Am. Chem. Soc. 2016, 138, 1674516753.

[14] Wrede, S.; Tian, H. Towards sustainable and efficient p-type metal oxide semiconductor materials in dye-sensitised photocathodes for solar energy conversion Phys. Chem. Chem. Phys. 2020, 22, 13850-13861.

[15] Creissen, C. E.; Warnan, J.; Antón-García, D.; Farré, Y.; Odobel, F.; Reisner, E. Inverse Opal CuCrO2 Photocathodes for $\mathrm{H} 2$ Production Using Organic Dyes and a Molecular Ni Catalyst ACS Catal. 2019, 9, 9530-9538.

[16] D’Amario, L.; Boschloo, G.; Hagfeldt, A.; Hammarström, L. Tuning of Conductivity and Density of States of NiO Mesoporous Films Used in p-Type DSSCs J. Phys. Chem. C 2014, 118, 19556-19564. 
[17] Odobel, F.; Pellegrin, Y.; Gibson, E.; Hagfeldt, A.; Smeigh, A.; Hammarström, L. Recent advances and future directions to optimize the performances of p-type dye-sensitized solar cells Coord. Chem. Rev. 2012, 256, 2414-2423.

[18] Zhang, L.; Favereau, L.; Farré, Y.; Mijangos, E.; Pellegrin, Y.; Blart, E.; Odobel, F.; Hammarström, L. Ultrafast and slow charge recombination dynamics of diketopyrrolopyrrole NiO dye sensitized solar cells Phys. Chem. Chem. Phys. 2016, 18, 18515-18527.

[19] Antila, L. J.; Ghamgosar, P.; Maji, S.; Tian, H.; Ott, S.; Hammarström, L. Dynamics and Photochemical H2 Evolution of Dye@NiO Photocathodes with a Biomimetic FeFeCatalyst ACS Energy Lett. 2016, 1, 1106-1111.

[20] Brown, A. M.; Antila, L. J.; Mirmohades, M.; Pullen, S.; Ott, S.; Hammarström, L. Ultrafast Electron Transfer Between Dye and Catalyst on a Mesoporous NiO Surface $J$. Am. Chem. Soc. 2016, 138, 8060-8063.

[21] Smeigh, A. L.; Pleux, L. L.; Fortage, J.; Pellegrin, Y.; Blart, E.; Odobel, F.; Hammarström, L. Ultrafast recombination for $\mathrm{NiO}$ sensitized with a series of perylene imide sensitizers exhibiting Marcus normal behaviour Chem. Commun. 2012, 48, 678-680.

[22] Zhang, X. L.; Huang, F.; Nattestad, A.; Wang, K.; Fu, D.; Mishra, A.; Baüerle, P.; Bach, U.; Cheng, Y.-B. Enhanced open-circuit voltage of p-type DSC with highly crystalline $\mathrm{NiO}$ nanoparticles Chem. Commun. 2011, 47, 4808-4810.

[23] Zhang, X. L.; Zhang, Z.; Chen, D.; Baüerle, P.; Bach, U.; Cheng, Y.-B. Sensitization of nickel oxide: improved carrier lifetime and charge collection by tuning nanoscale crystallinity Chem. Commun. 2012, 48, 9885-9887.

[24] Materna, K. L.; Beiler, A. M.; Thapper, A.; Ott, S.; Tian, H.; Hammarström, L. Understanding the Performance of NiO Photocathodes with Alkyl-Derivatized Cobalt Catalysts and a Push-Pull Dye ACS Appl. Mater. Interfaces 2020, 12, 31372-31381. 
[25] McCullough, S. M.; Evans, J. M.; Moot, T.; Taggart, A. D.; Troian-Gautier, L.; Cahoon, J. F. Cation Effects in p-Type Dye-Sensitized Solar Cells ACS Appl. Energy Mater. 2020, 3, 1496-1505.

[26] Pastore, M.; De Angelis, F. First-Principles Modeling of a Dye-Sensitized TiO2/IrO2 Photoanode for Water Oxidation J. Am. Chem. Soc. 2015, 137, 5798-5809.

[27] Umari, P.; Mosconi, E.; De Angelis, F. Relativistic GW calculations on CH3NH3PbI3 and CH3NH3SnI3 Perovskites for Solar Cell Applications Sci. Rep. 2014, 4, 4467.

[28] Preat, J.; Jacquemin, D.; Perpéte, E. A. Towards new efficient dye-sensitised solar cells Energy Environ. Sci. 2010, 3, 891-904.

[29] Martsinovich, N.; Troisi, A. Theoretical studies of dye-sensitised solar cells: from electronic structure to elementary processes Energy Environ. Sci. 2011, 4, 4473-4495.

[30] Powar, S.; Xiong, D.; Daeneke, T.; Ma, M. T.; Gupta, A.; Lee, G.; Makuta, S.; Tachibana, Y.; Chen, W.; Spiccia, L.; Cheng, Y.-B.; Götz, G.; Bäuerle, P.; Bach, U. Improved Photovoltages for p-Type Dye-Sensitized Solar Cells Using CuCrO2 Nanoparticles J. Phys. Chem. C 2014, 118, 16375-16379.

[31] Shao, Y.; de Ruiter, J. M.; de Groot, H. J. M.; Buda, F. Photocatalytic Water Splitting Cycle in a Dye-Catalyst Supramolecular Complex: Ab Initio Molecular Dynamics Simulations J. Phys. Chem. C 2019, 123, 21403-21414.

[32] Spies, J. A.; Perets, E. A.; Fisher, K. J.; Rudshteyn, B.; Batista, V. S.; Brudvig, G. W.; Schmuttenmaer, C. A. Collaboration between experiment and theory in solar fuels research Chem. Soc. Rev. 2019, 48, 1865-1873.

[33] Duchanois, T.; Liu, L.; Pastore, M.; Monari, A.; Cebrián, C.; Trolez, Y.; Darari, M.; Magra, K.; Francés-Monerris, A.; Domenichini, E.; et al. NHC-Based Iron Sensitizers for DSSCs Inorganics 2018, 6, 63.

[34] Pastore, M. First Principle Modelling of Materials and Processes in Dye-Sensitized Photoanodes for Solar Energy and Solar Fuels Computat. 2017, 5, 5. 
[35] Lucarini, F.; Fize, J.; Morozan, A.; Marazzi, M.; Natali, M.; Pastore, M.; Artero, V.; Ruggi, A. Insights into the mechanism of photosynthetic $\mathrm{H} 2$ evolution catalyzed by a heptacoordinate cobalt complex Sustain. Energy Fuels 2020, 4, 589-599.

[36] Piccinin, S.; Rocca, D.; Pastore, M. Role of Solvent in the Energy Level Alignment of Dye-Sensitized NiO Interfaces J. Phys. Chem. C 2017, 121, 22286-22294.

[37] Wykes, M.; Odobel, F.; Adamo, C.; Ciofini, I.; Labat, F. Anchoring groups for dyes in p-DSSC application: insights from DFT J. Mol. Model. 2016, 22, 289.

[38] Muñoz García, A. B.; Pavone, M. Structure and energy level alignment at the dyeelectrode interface in p-type DSSCs: new hints on the role of anchoring modes from ab initio calculations Phys. Chem. Chem. Phys. 2015, 17, 12238-12246.

[39] Massin, J.; Lyu, S.; Pavone, M.; Muñoz García, A. B.; Kauffmann, B.; Toupance, T.; Chavarot-Kerlidou, M.; Artero, V.; Olivier, C. Design and synthesis of novel organometallic dyes for $\mathrm{NiO}$ sensitization and photo-electrochemical applications Dalton Trans. 2016, 45, 12539-12547.

[40] Naik, P.; Planchat, A.; Pellegrin, Y.; Odobel, F.; Vasudeva Adhikari, A. Exploring the application of new carbazole based dyes as effective p-type photosensitizers in dyesensitized solar cells Sol. Energy 2017, 157, 1064 - 1073.

[41] Sun, L. L.; Zhang, T.; Wang, J.; Li, H.; Yan, L. K.; Su, Z. M. Exploring the influence of electron donating/withdrawing groups on hexamolybdate-based derivatives for efficient p-type dye-sensitized solar cells (DSSCs) RSC Adv. 2015, 5, 39821-39827.

[42] Zhang, T.; Guan, W.; Yan, L.; Ma, T.; Wang, J.; Su, Z. Theoretical studies on POM-based organic-inorganic hybrids containing double $\mathrm{D}-\mathrm{A} 1-\pi-\mathrm{A} 2$ chains for high-performance p-type, dye-sensitized solar cells (DSSCs) Phys. Chem. Chem. Phys. 2015, 17, 54595465 . 
[43] Mori, S.; Fukuda, S.; Sumikura, S.; Takeda, Y.; Tamaki, Y.; Suzuki, E.; Abe, T. ChargeTransfer Processes in Dye-Sensitized NiO Solar Cells The J. Phys. Chem. C 2008, 112, 16134-16139.

[44] Gardner, J. M.; Beyler, M.; Karnahl, M.; Tschierlei, S.; Ott, S.; Hammarström, L. LightDriven Electron Transfer between a Photosensitizer and a Proton-Reducing Catalyst Coadsorbed to NiO J. Am. Chem. Soc. 2012, 134, 19322-19325.

[45] Morandeira, A.; Boschloo, G.; Hagfeldt, A.; Hammarström, L. Photoinduced Ultrafast Dynamics of Coumarin 343 Sensitized p-Type-Nanostructured NiO Films J. Phys. Chem. B 2005, 109, 19403-19410.

[46] Migani, A.; Mowbray, D. J.; Zhao, J.; Petek, H.; Rubio, A. Quasiparticle Level Alignment for Photocatalytic Interfaces J. Chem. Theory Comput. 2014, 10, 2103-2113.

[47] Pastore, M.; Fantacci, S.; De Angelis, F. Modeling Excited States and Alignment of Energy Levels in Dye-Sensitized Solar Cells: Successes, Failures, and Challenges $J$. Phys. Chem. C 2013, 117, 3685-3700.

[48] Zheng, H.; Govoni, M.; Galli, G. Dielectric-dependent hybrid functionals for heterogeneous materials Phys. Rev. Materials 2019, 3, 073803.

[49] Borlido, P.; Marques, M. A. L.; Botti, S. Local Hybrid Density Functional for Interfaces J. Chem. Theory Comput. 2018, 14, 939-947.

[50] Gerosa, M.; Gygi, F.; Govoni, M.; Galli, G. The role of defects and excess surface charges at finite temperature for optimizing oxide photoabsorbers Nat. Mater. 2018, 17.

[51] Akimov, A. V.; Prezhdo, O. V. The PYXAID Program for Non-Adiabatic Molecular Dynamics in Condensed Matter Systems J. Chem. Theory Comput. 2013, 11, 4959-4972

[52] Duncan, W. R.; Prezhdo, O. V. Theoretical Studies of Photoinduced Electron Transfer in Dye-Sensitized TiO2 Annu. Rev. Phys. Chem. 2007, 58, 143-184 
[53] Duncan, W. R.; Craig, C. F.; Prezhdo, O. V. Time-Domain ab Initio Study of Charge Relaxation and Recombination in Dye-Sensitized TiO2 J. Am. Chem. Soc. 2007, 27, $8528-8543$

[54] Jiang, X.; Zheng, Q.; Lan, Z.; Saidi, W. A.; Ren, X.; Zhao, J. Real-time GW-BSE investigations on spin-valley exciton dynamics in monolayer transition metal dichalcogenide Sci. Adv. 2021, 7

[55] Yu, M.; Doak, P.; Tamblyn, I.; Neaton, J. B. Theory of Covalent Adsorbate Frontier Orbital Energies on Functionalized Light-Absorbing Semiconductor Surfaces J. Phys. Chem. Lett. 2013, 4, 1701-1706.

[56] Verdi, C.; Mosconi, E.; De Angelis, F.; Marsili, M.; Umari, P. Alignment of energy levels in dye/semiconductor interfaces by $G W$ calculations: Effects due to coadsorption of solvent molecules Phys. Rev. B 2014, 90, 155410.

[57] Umari, P.; Giacomazzi, L.; De Angelis, F.; Pastore, M.; Baroni, S. Energy-level alignment in organic dye-sensitized TiO2 from GW calculations J. Chem. Phys. 2013, 139, 014709 .

[58] Patrick, C. E.; Giustino, F. Quantitative Analysis of Valence Photoemission Spectra and Quasiparticle Excitations at Chromophore-Semiconductor Interfaces Phys. Rev. Lett. 2012, 109, 116801.

[59] Ping, Y.; Rocca, D.; Galli, G. Electronic excitations in light absorbers for photoelectrochemical energy conversion: first principles calculations based on many body perturbation theory Chem. Soc. Rev. 2013, 42, 2437-2469.

[60] Onida, G.; Reining, L.; Rubio, A. Electronic excitations: density-functional versus many-body Green's-function approaches Rev. Mod. Phys. 2002, 74, 601-659.

[61] Hedin, L. New Method for Calculating the One-Particle Green's Function with Application to the Electron-Gas Problem Phys. Rev., 139, A796-A823. 
[62] Faber, C.; Duchemin, I.; Deutsch, T.; Blase, X. Many-body Green's function study of coumarins for dye-sensitized solar cells Phys. Rev. B 2012, 86, 155315.

[63] Jacquemin, D.; Duchemin, I.; Blase, X. Benchmarking the Bethe-Salpeter Formalism on a Standard Organic Molecular Set J. Chem. Theory Comput. 2015, 11, 3290-3304.

[64] Govoni, M.; Galli, G. Large Scale GW Calculations J. Chem. Theory Comput. 2015, 11, 2680-2696.

[65] Liu, P.; Kaltak, M.; Klimeš, J. c. v.; Kresse, G. Cubic scaling GW: Towards fast quasiparticle calculations Phys. Rev. B 2016, 94, 165109.

[66] Wilhelm, J.; Golze, D.; Talirz, L.; Hutter, J.; Pignedoli, C. A. Toward GW Calculations on Thousands of Atoms J. Phys. Chem. Lett. 2018, 9, 306-312.

[67] Gao, W.; Xia, W.; Gao, X.; Zhang, P. Speeding up GW Calculations to Meet the Challenge of Large Scale Quasiparticle Predictions Sci. Rep. 2016, 6, 36849.

[68] Haastrup, S.; Strange, M.; Pandey, M.; Deilmann, T.; Schmidt, P. S.; Hinsche, N. F.; Gjerding, M. N.; Torelli, D.; Larsen, P. M.; Riis-Jensen, A. C.; Gath, J.; Jacobsen, K. W.; Mortensen, J. J.; Olsen, T.; Thygesen, K. S. The Computational 2D Materials Database: high-throughput modeling and discovery of atomically thin crystals 2D Mater. 2018, 5, 042002.

[69] Hüser, F.; Olsen, T.; Thygesen, K. S. How dielectric screening in two-dimensional crystals affects the convergence of excited-state calculations: Monolayer $\mathrm{MoS}_{2}$ Phys. Rev. B 2013, 88, 245309.

[70] Qiu, D. Y.; da Jornada, F. H.; Louie, S. G. Optical Spectrum of $\mathrm{MoS}_{2}$ : Many-Body Effects and Diversity of Exciton States Phys. Rev. Lett. 2013, 111, 216805.

[71] Zhang, Y.; Xia, W.; Wu, Y.; Zhang, P. Prediction of MXene based 2D tunable band gap semiconductors: GW quasiparticle calculations Nanoscale 2019, 11, 3993-4000. 
[72] Kresse, G.; Furthmüller, J. Efficient iterative schemes for ab initio total-energy calculations using a plane-wave basis set Phys. Rev. B 1996, 54, 11169-11186.

[73] Kresse, G.; Furthmüller, J. Efficiency of ab-initio total energy calculations for metals and semiconductors using a plane-wave basis set Comput. Mater. Sci. 1996, 6, 15 - 50.

[74] Shishkin, M.; Kresse, G. Implementation and performance of the frequency-dependent GW method within the PAW framework Phys. Rev. B 2006, 74, 035101.

[75] Kresse, G.; Joubert, D. From ultrasoft pseudopotentials to the projector augmented-wave method Phys. Rev. B 1999, 59, 1758-1775.

[76] Hutter, J.; Iannuzzi, M.; Schiffmann, F.; VandeVondele, J. cp2k: atomistic simulations of condensed matter systems Wiley Interdiscip. Rev. Comput. Mol. Sci. 2014, 4, 15-25.

[77] Rinaldi-Montes, N.; Gorria, P.; Martínez-Blanco, D.; Fuertes, A. B.; Puente-Orench, I.; Olivi, L.; Blanco, J. A. Size effects on the Néel temperature of antiferromagnetic NiO nanoparticles AIP Adv. 2016, 6, 056104.

[78] Cococcioni, M.; de Gironcoli, S. Linear response approach to the calculation of the effective interaction parameters in the LDA + U method Phys. Rev. B 2005, 71, 035105.

[79] Hybertsen, M. S.; Louie,S. G. First-Principles Theory of Quasiparticles: Calculation of Band Gaps in Semiconductors and Insulators Phys. Rev. Lett. 1985, 13, 1418-1421.

[80] Hybertsen, M. S.; Louie,S. G. Electron correlation in semiconductors and insulators: Band gaps and quasiparticle energies Phys. Rev. B 1986, 8, 5390-5413.

[81] Bruneval, F.; Marques, M. A. L. Benchmarking the Starting Points of the GW Approximation for Molecules J. Chem. Theory Comput. 2013, 9, 324-329.

[82] Jiang, H.; Gomez-Abal, R. I.; Rinke, P.; Scheffler, M. First-principles modeling of localized $d$ states with the $G W @$ LDA $+U$ approach Phys. Rev. B 2010, 82, 045108.

[83] Gajdoš, M.; Hummer, K.; Kresse, G.; Furthmüller, J.; Bechstedt, F. Linear optical properties in the projector-augmented wave methodology Phys. Rev. B 2006, 73, 045112. 
[84] Neugebauer, J.; Scheffler, M. Adsorbate-substrate and adsorbate-adsorbate interactions of Na and K adlayers on Al(111) Phys. Rev. B 1992, 46, 16067-16080.

[85] Mercurio, G.; McNellis, E. R.; Martin, I.; Hagen, S.; Leyssner, F.; Soubatch, S.; Meyer, J.; Wolf, M.; Tegeder, P.; Tautz, F. S.; Reuter, K. Structure and Energetics of Azobenzene on Ag(111): Benchmarking Semiempirical Dispersion Correction Approaches Phys. Rev. Lett. 2010, 104, 036102.

[86] Tonigold, K.; Groß, A. Dispersive interactions in water bilayers at metallic surfaces: A comparison of the PBE and RPBE functional including semiempirical dispersion corrections J. Comput. Chem. 2012, 33, 695-701.

[87] Grimme, S.; Antony, J.; Ehrlich, S.; Krieg, H. A consistent and accurate ab initio parametrization of density functional dispersion correction (DFT-D) for the 94 elements H-Pu J. Chem. Phys. 2010, 132, 154104.

[88] Bussi, G.; Donadio, D.; Parrinello, M. Canonical sampling through velocity rescaling J. Chem. Phys. 2007, 126, 014101.

[89] Kurmaev, E. Z.; Wilks, R. G.; Moewes, A.; Finkelstein, L. D.; Shamin, S. N.; Kuneš, J. Oxygen x-ray emission and absorption spectra as a probe of the electronic structure of strongly correlated oxides Phys. Rev. B 2008, 77, 165127.

[90] Hüfner, S. Electronic structure of $\mathrm{NiO}$ and related $3 \mathrm{~d}$-transition-metal compounds $A d v$. Phys. 1994, 43, 183-356.

[91] Powell, R. J.; Spicer, W. E. Optical Properties of NiO and $\mathrm{CoO}$ Phys. Rev. B 1970, 2, 2182-2193.

[92] Dong, T.; Suk, H.; Hosun, H. Optical Properties of Black NiO and CoO Single Crystals Studied with Spectroscopic Ellipsometry J. Korean Phys. Soc. 2007, 50, 632.

[93] Sawatzky, G. A.; Allen, J. W. Magnitude and Origin of the Band Gap in NiO Phys. Rev. Lett. 1984, 53, 2339-2342. 
[94] Bengone, O.; Alouani, M.; Hugel, J.; Blöchl, P. LDA+U calculated electronic and structural properties of $\mathrm{NiO}(001)$ and $\mathrm{NiO}(111) \mathrm{p}(2 \times 2)$ surfaces Comput. Mater. Sci. 2002, 24, $192-198$.

[95] Kobayashi, S.; Nohara, Y.; Yamamoto, S.; Fujiwara, T. GW approximation with LSDA+ $\mathrm{U}$ method and applications to $\mathrm{NiO}, \mathrm{MnO}$, and $\mathrm{V}_{2} \mathrm{O}_{3}$ Phys. Rev. B 2008, 78, 155112.

[96] Rödl, C.; Fuchs, F.; Furthmüller, J.; Bechstedt, F. Quasiparticle band structures of the antiferromagnetic transition-metal oxides $\mathrm{MnO}, \mathrm{FeO}, \mathrm{CoO}$, and $\mathrm{NiO}$ Phys. Rev. B 2009, $79,235114$.

[97] Li, J.-L.; Rignanese, G.-M.; Louie, S. G. Quasiparticle energy bands of $\mathrm{NiO}$ in the $G W$ approximation Phys. Rev. B 2005, 71, 193102.

[98] Faleev, S. V.; van Schilfgaarde, M.; Kotani, T. All-Electron Self-Consistent GW Approximation: Application to Si, MnO, and NiO Phys. Rev. Lett. 2004, 93, 126406.

[99] Onida, G.; Reining, L.; Godby, R. W.; Del Sole, R.; Andreoni, W. Ab Initio Calculations of the Quasiparticle and Absorption Spectra of Clusters: The Sodium Tetramer Phys. Rev. Lett. 1995, 75, 818-821.

[100] Cinquini, F.; Giordano, L.; Pacchioni, G.; Ferrari, A. M.; Pisani, C.; Roetti, C. Electronic structure of $\mathrm{NiO} / \mathrm{Ag}(100)$ thin films from DFT+U and hybrid functional DFT approaches Phys. Rev. B 2006, 74, 165403.

[101] Ferrari, A. M.; Pisani, C.; Cinquini, F.; Giordano, L.; Pacchioni, G. Cationic and anionic vacancies on the $\mathrm{NiO}(100)$ surface: $\mathrm{DFT}+\mathrm{U}$ and hybrid functional density functional theory calculations J. Chem. Phys. 2007, 127, 174711.

[102] Momida, H.; Oguchi, T. First-Principles Studies of Antiferromagnetic MnO and $\mathrm{NiO}$ Surfaces J. Phys. Soc. Jpn. 2003, 72, 588-593.

[103] Irwin, M. D.; Buchholz, D. B.; Hains, A. W.; Chang, R. P. H.; Marks, T. J. p-Type semiconducting nickel oxide as an efficiency-enhancing anode interfacial layer in polymer bulk-heterojunction solar cells Proc. Natl. Acad. Sci. U.S.A. 2008, 105, 2783-2787. 
[104] Janak, J. F. Proof that $\frac{\partial E}{\partial n_{i}}=\varepsilon$ in density-functional theory Phys. Rev. B 1978, 18, 71657168.

[105] Szuber, J. Electronic properties of the $\mathrm{NiO}(100)$ surface after thermal cleaning in ultrahigh vacuum J. Electron Spectrosc. Relat. Phenom. 1984, 34, 337 - 341.

[106] Tench, D. M.; Yeager, E. Capacitance Measurements on Lithiated Nickel Oxide Electrodes J. Electrochem. Soc. 1973, 120, 164.

[107] Madjid, A. H.; Martinez, J. M. Thermionic Emission from Nickel Oxide Phys. Rev. Lett. 1972, 28, 1313-1315.

[108] Hinuma, Y.; Grüneis, A.; Kresse, G.; Oba, F. Band alignment of semiconductors from density-functional theory and many-body perturbation theory Phys. Rev. B 2014, 90, 155405.

[109] Correa, N. M.; Levinger, N. E. What Can You Learn from a Molecular Probe? New Insights on the Behavior of C343 in Homogeneous Solutions and AOT Reverse Micelles J. Phys. Chem. B 2006, 110, 13050-13061.

[110] Zhu, L.; Tu, Z.; Yi, Y.; Wei, Z. Achieving Small Exciton Binding Energies in Small Molecule Acceptors for Organic Solar Cells: Effect of Molecular Packing J. Phys. Chem. Lett. 2019, 10, 4888-4894.

[111] Dualeh, A.; De Angelis, F.; Fantacci, S.; Moehl, T.; Yi, C.; Kessler, F.; Baranoff, E.; Nazeeruddin, M. K.; Grätzel, M. Influence of Donor Groups of Organic D- $\pi$-A Dyes on Open-Circuit Voltage in Solid-State Dye-Sensitized Solar Cells J. Phys. Chem. C 2012, $116,1572-1578$.

[112] Xu, M.; Zhang, M.; Pastore, M.; Li, R.; De Angelis, F.; Wang, P. Joint electrical, photophysical and computational studies on D- $\pi$-A dye sensitized solar cells: the impacts of dithiophene rigidification Chem. Sci. 2012, 3, 976-983. 
[113] Garcia-Lastra, J. M.; Rostgaard, C.; Rubio, A.; Thygesen, K. S. Polarization-induced renormalization of molecular levels at metallic and semiconducting surfaces Phys. Rev. B 2009, 80, 245427.

[114] Neaton, J. B.; Hybertsen, M. S.; Louie, S. G. Renormalization of Molecular Electronic Levels at Metal-Molecule Interfaces Phys. Rev. Lett. 2006, 97, 216405.

[115] Garcia-Lastra, J. M.; Thygesen, K. S. Renormalization of Optical Excitations in Molecules near a Metal Surface Phys. Rev. Lett. 2011, 106, 187402.

[116] Golze, D.; Dvorak, M.; Rinke, P. The GW Compendium: A Practical Guide to Theoretical Photoemission Spectroscopy Front. Chem. 2019, 7, 377.

[117] Di Valentin, C.; Botti, S.; Cococcioni, M. First Principles Approaches to Spectroscopic Properties of Complex Materials [E-Book]; Top. Curr. Chem. ;; Springer: Berlin, Heidelberg, 2014.

[118] De Angelis, F.; Fantacci, S.; Gebauer, R. Simulating Dye-Sensitized TiO2 Heterointerfaces in Explicit Solvent: Absorption Spectra, Energy Levels, and Dye Desorption $J$. Phys. Chem. Lett. 2011, 2, 813-817.

[119] Bella, F.; Gerbaldi, C.; Barolo, C.; Grätzel, M. Aqueous dye-sensitized solar cells Chem. Soc. Rev. 2015, 44, 3431-3473.

[120] Zhao, W.; Bajdich, M.; Carey, S.; Vojvodic, A.; Nørskov, J. K.; Campbell, C. T. Water Dissociative Adsorption on $\mathrm{NiO}(111)$ : Energetics and Structure of the Hydroxylated Surface ACS Catal. 2016, 6, 7377-7384.

[121] Refson, K.; Wogelius, R. A.; Fraser, D. G.; Payne, M. C.; Lee, M. H.; Milman, V. Water chemisorption and reconstruction of the MgO surface Phys. Rev. B 1995, 52, $10823-$ 10826.

[122] Hosseinpour, S.; Tang, F.; Wang, F.; Livingstone, R. A.; Schlegel, S. J.; Ohto, T.; Bonn, M.; Nagata, Y.; Backus, E. H. G. Chemisorbed and Physisorbed Water at the TiO2/Water Interface J. Phys. Chem. Lett. 2017, 8, 2195-2199. 
[123] Galliano, S.; Bella, F.; Gerbaldi, C.; Falco, M.; Viscardi, G.; Grätzel, M.; Barolo, C. Photoanode/Electrolyte Interface Stability in Aqueous Dye-Sensitized Solar Cells Energy Technol. 2017, 5, 300-311.

[124] Boschloo, G.; Hagfeldt, A. Spectroelectrochemistry of Nanostructured NiO J. Phys. Chem. B 2001, 105, 3039-3044.

[125] Lichterman, M. F.; Hu, S.; Richter, M. H.; Crumlin, E. J.; Axnanda, S.; Favaro, M.; Drisdell, W.; Hussain, Z.; Mayer, T.; Brunschwig, B. S.; Lewis, N. S.; Liu, Z.; Lewerenz, H.-J. Direct observation of the energetics at a semiconductor/liquid junction by operando X-ray photoelectron spectroscopy Energy Environ. Sci. 2015, 8, 2409-2416.

[126] Schwanitz, K.; Weiler, U.; Hunger, R.; Mayer, T.; Jaegermann, W. Synchrotron-Induced Photoelectron Spectroscopy of the Dye-Sensitized Nanocrystalline TiO2/Electrolyte Interface: Band Gap States and Their Interaction with Dye and Solvent Molecules The J. Phys. Chem. C 2007, 111, 849-854.

[127] Hahlin, M.; Johansson, E. M. J.; Plogmaker, S.; Odelius, M.; Hagberg, D. P.; Sun, L.; Siegbahn, H.; Rensmo, H. Electronic and molecular structures of organic dye/TiO2 interfaces for solar cell applications: a core level photoelectron spectroscopy study Phys. Chem. Chem. Phys. 2010, 12, 1507-1517. 